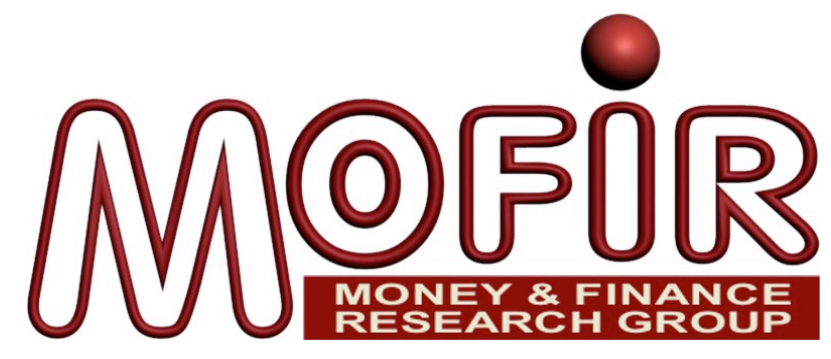

\title{
CREdit CRunches, Asset Prices AND TECHNOLOGICAL CHANGE
}

\author{
LUIS ARAUJO \\ Michigan State University \\ Sao Paolo School of Economics - FGV \\ RaOUl MinetTi \\ Michigan State University
}

MoFiR working paper $n^{\circ} 61$

March 2012 


\title{
Credit Crunches, Asset Prices and Technological Change
}

\author{
Luis Araujo \\ Michigan State University and São Paulo School of Economics-FGV \\ Raoul Minetti* \\ Michigan State University
}

\begin{abstract}
We investigate the effects of a credit crunch in an economy where firms can operate a mature technology or restructure their activity and adopt a new technology. We show that firms' collateral and credit relationships ease firms' access to credit and investment but can also inhibit firms' restructuring. When this occurs, negative collateral or productivity shocks and the resulting drop in the price of collateral assets squeeze collateral-poor firms out of the credit market but foster the restructuring of collateral-rich firms. We characterize conditions under which such an increase in firms' restructuring occurs within existing credit relationships or through their breakdown. The analysis reveals that the credit and asset market policies adopted during the recent credit crunch can promote investment but might also slow down a process of Shumpeterian restructuring in the credit market.
\end{abstract}

Keywords: Aggregate Restructuring, Collateral, Credit Relationships, Credit Crunch.

JEL Codes: E44.

\section{Introduction}

The 2008-2010 financial crisis has put the spotlight on the role of the credit market in firms' investment decisions. During the crisis, a major decline in the value of collateral assets, especially real estate, has put credit relationships between firms and lenders under strain, allegedly resulting into a drop in total credit and investment. The literature offers well-established theoretical arguments for interpreting these effects of a credit crunch. When entrepreneurs cannot fully commit to repay their lenders, the availability of collateralizable assets eases their access to credit (Kiyotaki and Moore, 1997). Ex post, after entrepreneurs default, lenders can repossess collateral and this compensates for the limited pledgeability of entrepreneurs' output; ex ante, lenders' threat to repossess collateral deters entrepreneurs' misbehavior. Credit relationships can enhance these benefits of collateral. For example, lenders who establish informationally intensive

${ }^{*}$ Department of Economics, Michigan State University. E-mail: minetti@msu.edu. Phone: +1-517-355-7349. Address: 101 Marshall Hall, East Lansing, MI 48824-1038, USA. We wish to thank Satyajit Chatterjee, Luigi Guiso, Maurizio Iacopetta, Matteo Iacoviello, Rowena Pecchenino, Susan Chun Zhu and seminar participants at Bank of Italy/Ente Einaudi, Boston College, Central Michigan University, London School of Economics (FMG/ESRC Conference), Michigan State University, Stockholm School of Economics, Universidad Carlos III (Madrid), University of Michigan at Ann Arbor, Western Michigan University, the Federal Reserve Bank of Chicago "Summer Workshop on Money, Banking, Payments and Finance", Midwest Economic Theory Meetings, Midwest Macroeconomics Meetings, the 2011 OFCE-SKEMA Workshop on Models with Credit Frictions and Money (Nice). All remaining errors are ours. 
relationships with entrepreneurs can better monitor their assets and, hence, recover more value from asset repossession (Diamond and Rajan, 2001). In an aggregate perspective, an implication of these arguments is that shocks that erode the value of collateral assets or break credit relationships depress total investment by hindering firms' access to external finance (Kiyotaki and Moore, 1997; Holmstrom and Tirole, 1997).

While useful to explain key mechanisms of transmission of the crisis, these arguments yield limited insights into the effects of a credit crunch on technological change. If one allows for technological change, two questions arise naturally: Do collateral and credit relationships ease entrepreneurs' restructuring activity, meant as the upgrade from mature technologies to new ones? And therefore, in an aggregate perspective, do shocks that erode the value of collateral and break credit relationships depress aggregate restructuring as they allegedly depress investment? This paper takes a step towards addressing these questions. We study an economy where entrepreneurs operate a mature technology or restructure their activity and adopt a new technology. Lenders, in turn, acquire information that is essential for repossessing and liquidating productive assets pledged as collateral when entrepreneurs default (as in Diamond and Rajan, 2001, for example). Lenders' information on collateral assets eases entrepreneurs' access to credit. However, their information renders lenders reluctant to finance entrepreneurs' restructuring activity. In fact, the new technology has less assets pledgeable as collateral. Furthermore, the information on the assets of the mature technology is at least partially specific and non-transferable to the collateral assets of the new technology. Therefore, expecting that the information they have accumulated on mature collateral assets will go wasted if entrepreneurs upgrade to the new technology, lenders may hinder entrepreneurs' restructuring efforts.

In this economy, entrepreneurs can form informationally intensive credit relationships with lenders to transfer them more information on collateral assets and obtain cheaper financing. Yet, the information accumulated within the relationships exacerbates lenders' incentive to inhibit firms' restructuring. When technological inertia arises, entrepreneurs can break their credit relationships and restructure. However, this wastes the information accumulated. Hence, these relationships and technological inertia can be long-lasting. The resulting distribution of firms across collateral values replicates salient features of that obtained in previous general equilibrium models of the credit market (e.g., Holmstrom and Tirole, 1997). Collateral-poor firms lack access to credit because they cannot pledge enough expected returns to lenders, even when these obtain high quality information on collateral assets. Furthermore, firms with medium collateral value obtain credit from informed (relationship) lenders. The novelty consists of firms' technology adoption. While firms with medium collateral value potentially restructure, collateral-rich firms with credit relationships preserve the mature technology. In fact, their lenders expect a large depreciation in the value of their information if the mature technology is abandoned in favor of the new technology.

We study the effects of contractions in the value of collateral assets and in productivity. Consider collateral shocks (the reasoning for productivity shocks is similar). Following the drop in the price of collateral assets, the credit relationships of collateral-poor firms break down because these firms can no longer pledge enough expected returns to lenders. The exclusion of collateral-poor firms from the credit market tends to reduce 
both total investment and aggregate restructuring. Consider next collateral-rich firms. The reduction in the asset price erodes the value of the information acquired by their lenders on mature collateral assets. This mitigates lenders' technological inertia, allowing restructuring to occur within the relationships. This also increases the incentive of collateral-rich firms to deliberately break their credit relationships, borrow from new lenders and restructure. These effects work in the direction of fostering the restructuring of collateral-rich firms. If the increase in the restructuring of collateral-rich firms outweighs the drop in the restructuring of collateral-poor firms, the shock will cause a decline in total investment but an increase in total restructuring.

What are the consequences of the increased restructuring of collateral-rich firms for the credit market? There is a credit regime in which lenders' technological inertia is weak and/or firms derive large benefits from relationships: in this regime, collateral-rich firms restructure within their relationships. There is instead a credit regime in which lenders' technological inertia is strong and/or firms derive small benefits from relationships: in this regime, collateral-rich firms restructure by breaking their relationships and borrowing from new lenders. Thus, depending on the credit regime, the increase in restructuring activity induced by the shock can entail a moderate or a major breakdown of credit relationships. This is also important for the impact of the shock on output, because the breakdown of relationships can depress output by raising asset liquidation costs.

In the last part of the paper, we investigate the effects of two unconventional policies carried out during the financial crisis: an intervention in the collateral asset market aimed at sustaining the asset price after the shock and a policy of direct lending to collateral-poor firms. We find that both policies foster total investment but may dampen the increase in the restructuring of collateral-rich firms during a credit crunch. The case of the direct lending policy is especially insightful. In our economy, the credit rationing of collateral-poor firms following the shock fosters the restructuring of collateral-rich firms by bringing down collateral asset demand and prices. A policy of direct lending dampens this effect.

This paper especially relates to two strands of literature. The first investigates the impact of a disruption in the financial structure on aggregate investment (for recent studies, see, e.g., Gertler and Karadi, 2010, and Gertler and Kiyotaki, 2010). We have discussed some key elements we share with Holmstrom and Tirole (1997). We also borrow properties of our modelling strategy from their paper, such as the focus on a finite horizon economy. Den Haan, Ramey and Watson (2003) and dell'Ariccia and Garibaldi (2001) are other related papers in this strand of literature. These studies analyze the breakdown of credit relationships that can be caused by a recession in economies with search frictions. While in these studies a breakdown of credit relationships depresses investment, in our economy it depresses investment but may also foster aggregate restructuring.

The second strand of literature analyzes the impact of recessions on firms' restructuring. Most of this literature neglects the role of the credit market for aggregate restructuring. Caballero and Hammour (2004), Ramey (2004) and Barlevy (2003) are exceptions. These studies show that credit frictions can become more severe during recessions, hindering aggregate restructuring. Caballero and Hammour (2004) show 
that, because of credit frictions, production units can be destroyed at an excessive rate during a recession. Furthermore, during the following recovery, the creation of new production units can be too slow and most of the recovery can occur via a slowdown of destruction. Ramey (2004) endogenizes financial managers' project selection and shows that, if managers have empire-building incentives, during recessions they can discard efficient projects to preserve the size of their portfolios. Barlevy (2003) finds that credit frictions can reverse the "cleansing effect" of recessions by leading to the disruption of high-surplus production units rather than low-surplus ones. This paper endorses a view opposite to all these studies: while it negatively affects investment, the breakdown of informationally intensive credit relationships also mitigates lenders' technological inertia.

The remainder of the paper is organized as follows. In Section 2, we outline and discuss the setup. Section 3 solves for the equilibrium. In Section 4, we investigate the effects of shocks. Section 5 analyzes the robustness of the analysis. Section 6 considers the effect of policies in the asset and credit markets. Section 7 concludes. The Appendix contains the main proofs while more technical proofs are relegated to a Supplement.

\section{The Model}

This section describes the setup of the model. Table 1 summarizes the notation while Figure 1 illustrates the timing of events.

\subsection{Agents, Goods and Technology}

Consider a four-date economy $(t=0,1,2,3)$ populated by a unit continuum of entrepreneurial firms and a continuum of investors of measure larger than one. There is a final consumption good, which can be produced and stored, and productive assets of two vintages, mature and new. Entrepreneurs have no endowment while each investor is initially endowed with an amount $\omega$ of final good. All agents are risk neutral and consume on date 3 .

Each entrepreneur can implement one indivisible project. On date 2, an entrepreneur can experience a technological innovation. If the innovation occurs, the entrepreneur chooses whether to restructure his activity, adopting a new technology, or operate a mature, less productive technology. In the innovation does not occur, the entrepreneur has to operate the mature technology. Under the mature (new) technology, on date 3 the entrepreneur transforms an amount $i<\omega$ of final good into one unit of mature (new) assets. With probability $\pi>1 / 2$ the project succeeds and the mature (new) assets yield an output $y(y(1+n))$ of final good; otherwise the project fails and the entrepreneur goes out of business. In this case, a fraction $a$ $(\phi a)$ of mature (new) assets can be redeployed outside the firm. a captures the amount of collateralizable assets of an entrepreneur and is uniformly distributed across entrepreneurs over the domain $[0,1] . \phi \leq 1$ is a parameter that reflects the redeployability of new assets relative to mature assets. 


\begin{tabular}{|c|c|c|c|}
\hline Date 0 & Date 1 & Date 2 & Date 3 \\
\hline $\begin{array}{l}\text { Entrepreneurs form } \\
\text { credit relationships } \\
\text { with investors }\end{array}$ & $\begin{array}{l}\text { Entrepreneurs and } \\
\text { investors write credit } \\
\text { contracts }\end{array}$ & $\begin{array}{l}\text { - Lenders carry out } \\
\text { actions necessary } \\
\text { for innovations } \\
\text { - Innovations occur }\end{array}$ & $\begin{array}{l}\text { - Entrepreneurs carry out } \\
\text { projects } \\
\text { - Projects succeed or assets } \\
\text { are liquidated } \\
\text { - Agents consume }\end{array}$ \\
\hline
\end{tabular}

Figure 1: Timing of Events.

On date 3, each entrepreneur still in business can reuse one unit of liquidated assets, obtaining an amount $\eta \theta$ of final good. $\theta$ is uniformly distributed across entrepreneurs over the domain $[0, \bar{\theta}] ; \eta$ represents the aggregate productivity of liquidated assets. ${ }^{1}$

\subsection{Credit Sector}

Each entrepreneur can write a credit contract with one investor on date 1. A lender interacts with an entrepreneur along two dimensions besides credit provision: she exerts control and she acquires information. Following an established literature (e.g., Aghion and Bolton, 1992; Rajan, 1992), we allow the lender to exert control over production opportunities. Precisely, on date 2 the lender can carry out a costless action that affects the probability of the innovation: if she carries out this action, the innovation will occur with probability $1-\sigma(0<\sigma<1)$; otherwise, the innovation cannot occur.

The lender also acquires information as a by-product of her financing activity. As in Diamond and Rajan (2001) and Habib and Jonsen (1999), this information enables her to obtain more than other agents from the liquidation of the entrepreneur's assets, that is, the lender "monitors" collateralizable assets. Precisely, the share of liquidation value that the lender recovers equals the amount of her information on the assets; the rest of the liquidation value is lost in the form of a (nominal or real) transaction cost. By contrary, we normalize to zero the net amount of final good that any other agent obtains from asset liquidation.

Having characterized the nature of information, we have to specify its amount. We allow the entrepreneur to influence this amount by choosing the type of funding, relationship or transactional. Precisely, on date 0 each entrepreneur chooses whether to establish an informationally intensive credit relationship with his financier on date 1 or obtain a transactional loan. Consider first mature assets: $\sigma \mu$ (respectively, $\mu$ ) is the amount of information of a lender if she does (not) carry out the action for the innovation; furthermore, for a relationship lender $\mu=M$ while for a transactional lender $\mu=m$. This specification has two features. First, in an informationally intensive credit relationship a lender acquires more information about the entrepreneur's

\footnotetext{
${ }^{1}$ Building on the analysis of collateral asset markets in Shleifer and Vishny (1992), we generate a downward sloping demand for collateral assets by allowing for heterogeneity in entrepreneurs' ability to reuse assets.
} 
TABLE I.

Notation of the Model.

\begin{tabular}{ll}
\hline \hline Probability of project success & $\pi$ \\
Output of mature technology & $y$ \\
Productivity edge of new technology over mature & $n$ \\
Collateral assets of firm & $a$ \\
Investment requirement of project & $i$ \\
Share of verifiable output & $l$ \\
Probability of innovation & $1-\sigma$ \\
Aggregate productivity liquidated assets & $\eta$ \\
Idiosyncratic productivity liquidated assets & $\theta$ \\
Relative redeployability new assets & $\phi$ \\
Information of (share of asset rescued by) relationship lender & $M$ \\
Information of (share of asset rescued by) transactional lender & $m$ \\
\hline \hline
\end{tabular}

Note. The table summarizes the symbols used in the model (second column) and their description (first column).

assets $(M>m)$. Second, when a lender allows the innovation, she acquires less information on mature assets $(\sigma<1) .^{2}$ This reflects the idea that the lender has less opportunities - and with endogenous information acquisition, less incentives - to acquire information on a technology if the entrepreneur is working to abandon it. Consider next new assets: denoting the amount of information by $\mu_{n}$, we let $\mu_{n} \phi=0$. Thus, a lender recovers less value from liquidating new assets than from liquidating mature assets - the normalization to zero is for simplicity.

\subsection{Contractual Structure}

As in Aghion and Bolton (1992) and Diamond and Rajan (2001), a lender cannot contractually commit to carry out her action necessary for the innovation because this action is non-verifiable; moreover, imperfect enforceability limits agents' commitment to pecuniary transfers and, hence, the design of pecuniary incentives for the lender. Specifically, in the event of project success, only a fraction $l$ of the output is verifiable while the rest accrues privately to the entrepreneur. In the event of project failure and asset liquidation, the lender cannot commit the specific liquidation skills tied to her information about the assets. Thus, as in Diamond and Rajan (2001), she can threaten to withhold her skills during the liquidation, forcing a renegotiation of the allocation of the asset liquidation proceeds to appropriate them in full. ${ }^{3}$

\subsection{Discussion}

In the real sector, the difference between the two technologies is that the new technology yields more output $(y(1+n)>y)$ but its assets have lower liquidation value $\left(\mu_{n} \phi p a<\mu p a\right.$, where $p$ denotes the market price

\footnotetext{
${ }^{2}$ Letting the amount of information aquired by the lender on the mature technology be equal to the probability that the mature technology is adopted simplifies the algebra but is not relevant for the results.

${ }^{3}$ As in Diamond and Rajan (2001), for simplicity the lender has all the bargaining power in the renegotiation.
} 
of assets). We put forward two interpretations. First, new technologies typically have less assets pledgeable as collateral, i.e., $\phi<1$ (Hall, 2001; Berlin and Butler, 2002; Rajan and Zingales, 2001). ${ }^{4}$ Carpenter and Petersen (2002) argue that "high-tech investments have limited collateral value. R\&D investment, which is predominantly salary payments, has little salvage value in the event of failure. Furthermore, physical investments designed to embody $R \& D$ results are likely to be firm specific, and therefore may have little collateral value". Second, for a given liquidation value, lenders typically have less experience in liquidating new vintages of assets than mature ones, i.e., $\mu_{n}<\mu$.

In the credit sector, there are three features worth discussion: the control exerted by a lender; the characterization of information as asset liquidation skills; the amount of information of a lender. We share with Aghion and Bolton (1992) and Rajan (1992), for example, the assumption that a lender carries out an interim action that affects production opportunities. This action has several real world counterparts. It can consist of providing the entrepreneur with advice or information for expanding the firm's technological frontier; in an R\&D race, it can consist of concealing the findings of the entrepreneur's internal research from her competitors (Bhattacharya and Chiesa, 1995); if the lender has representatives on the board of the firm, as in the case of German and Japanese banks, it can consist of voting for an innovative strategy. In other circumstances, this action can consist of a refinancing (Rajan, 1992): the need for refinancing is likely for a new technology which generally yields little interim cash flow, especially at the R\&D stage (Goodakre and Tonks, 1995). Aghion and Bolton (1992) discuss examples of other actions of lenders which can affect innovation opportunities, such as supporting firms' mergers and spin-offs.

We borrow the characterization of information as asset liquidation skills from Diamond and Rajan (2001) and Habib and Jonsen (1999). The critical feature is that the lender acquires more information than the entrepreneur and the other investors. As for latter, following Diamond and Rajan (2001), our assumption reflects the idea that the lender obtains more information on collateralizable assets through her financing activity. As for the former, "Because he [the entrepreneur] is a specialist at maximizing the value of the asset in its primary use [...] it is reasonable to assume that he lacks the skill even to identify the asset's next best use or to recognize clearly the occurrence of the bad states, in which case he risks maintaining it in a suboptimal use" (Habib and Johnsen, 1999, p. 145).

The second feature of our information structure is the amount of information. In several models, the distinct characteristic of a relationship lender is her informational advantage over a transactional lender (Berger and Udell, 1998). Indeed, Berger and Udell argue that "banks may acquire private information over the course of a relationship" (1995, p. 352) and "under relationship lending, banks acquire information over time through contact with the firm, its owner, and its local community" (2002, p. 32). ${ }^{5}$

\footnotetext{
${ }^{4}$ In the model, we normalize to zero the value that a lender obtains from the liquidation of a new asset. We could allow new assets to have positive liquidation value, though lower than that of mature assets $\left(0<\mu_{n} \alpha_{n}<1\right)$. The results would carry through but the analysis would be more cumbersome.

${ }^{5}$ See also Guiso and Minetti (2010) for empirical evidence on this.
} 


\section{Equilibrium}

In solving for the equilibrium, we start with agents' decisions taking as given the market price of assets $p$. Next, we solve for the asset price. Finally, we characterize the distribution of firms across collateral values (a) according to whether a firm obtains credit or not, and, if it obtains credit, according to the type of funding it chooses (relationship or transactional) and the technology it adopts (mature or new). Throughout this section, we assume that $\phi=1$, that is, we let mature and new assets have the same redeployability in the asset market. This means that the difference between the return that a lender obtains from the liquidation of mature assets $(\mu p a)$ and the return she obtains from the liquidation of new assets $\left(\mu_{n} \phi p a\right)$ is fully attributable to her lower knowledge about the new assets $\left(\mu_{n}<\mu\right)$. Moreover, this implies that the market price of assets can be solved independently of the distribution of entrepreneurs between the mature and the new technology. In the Robustness analysis, we will consider the case in which $\phi<1$.

\subsection{Agents' Decisions}

We identify the possible outcomes of the interaction between a lender and an entrepreneur with the pair $(\mu, \alpha)$, where $\mu \in\{M, m\}$ denotes the behavior of the entrepreneur (i.e., whether the entrepreneur chooses relationship or transactional funding) and $\alpha \in\{A, \bar{A}\}$ denotes the behavior of the lender (i.e., whether the lender chooses to implement the action that allows the innovation). Moreover, we say that an outcome $(\mu, \alpha)$ is feasible if credit is available to fund such an outcome and there is a contract under $\mu$ that induces $\alpha$.

We focus first on the entrepreneurs who can obtain credit under both relationship and transactional funding and solve for agents' decisions by backward induction: first, we solve for a lender's action; then, we solve for an entrepreneur's choice between the two types of funding. Consider the date 2 lender's decision whether to carry out the action necessary for the innovation. The contractual structure implies that the contract only determines the loan granted and the repayment to the lender in the event of project success, contingent on the technology adopted. Denote by $r\left(r_{n}\right)$ the repayment contractually due to the lender if the mature (new) technology is successfully adopted. The lender compares her expected return if the innovation can occur with her expected return if the innovation cannot occur. Using simple algebra and assuming that she breaks a tie in favour of inaction, the lender will carry out the action if and only if

$$
r_{n}-r>\frac{(1+\sigma)(1-\pi) p a \mu}{\pi} .
$$

Inequality (1) is the lender's incentive compatibility constraint. The left hand side of (1) is the spread between the repayment in the event of successful adoption of the new technology and the repayment in the event of successful adoption of the mature technology. The right hand side of (1) is (a monotonic transformation of) the reduction in her liquidation proceeds that the lender suffers if the entrepreneur restructures and the project fails. This reduction, which is due to the lender's worse ability to liquidate new assets $\left(\mu_{n}=0\right)$, is positively related to the lender's information $\mu$ and to the liquidation value $p a$ of mature 
assets.

The lender will allow the innovation if and only if, as in (1), the contract guarantees her a sufficiently higher repayment if the new technology is successfully adopted, compensating her for the reduction in her expected liquidation proceeds. Lemma 1 characterizes the conditions under which there exist contracts that induce the lender to carry out the action necessary for the innovation.

LEMMA 1: There exist contracts that induce the lender to carry out the action necessary for the innovation if and only if the entrepreneurs's collateral assets satisfy $a<\bar{a}(p, \mu)$, where

$$
\bar{a}(p, \mu) \equiv \frac{\pi l y(1+n)-i}{p \sigma(1-\pi) \mu} .
$$

PROOF. In the Appendix.

The intuition behind Lemma 1 is as follows. The spread $r_{n}-r$ that can be specified in the contract is bounded. On the one hand, the repayment $r_{n}$ for the new technology is constrained above by the entrepreneur's limited liability constraint $r_{n} \leq l y(1+n)$. On the other hand, for a given $r_{n}$, the repayment $r$ for the mature technology is constrained below by the lender's participation constraint. ${ }^{6}$ Lemma 1 shows that if $a \geq \bar{a}(p, \mu)$, in (1) the left hand side falls short of the right hand side for any feasible pair $\left(r_{n}, r\right)$. In this region, the lender impedes the entrepreneur's restructuring activity.

Lemma 1 yields two insights. First, for a given market price of assets, the lender is more likely to induce technological inertia within a credit relationship, that is, $\bar{a}(p, M)<\bar{a}(p, m)$. In fact, because of her better information $(M>m)$, a relationship lender has greater ability to liquidate mature assets than a transactional lender, that is in (1) the right hand side is higher. Second, the lender is more likely to induce technological inertia when an entrepreneur is rich in collateral (has a high $a$ ) and when the market price of assets $p$ is higher. Intuitively, a lender loses more from the depreciation of her asset liquidation skills when these skills are better developed $(M>m)$ and the value $p a$ of the collateral assets of the entrepreneur is higher.

We can now solve for an entrepreneur's funding choice. Henceforth, we assume that an entrepreneur breaks a tie in favour of relationship funding.

LEMMA 2: An entrepreneur will choose transactional funding if and only if

$$
a \in[\bar{a}(p, M), \bar{a}(p, m)) \text { and } a<\widehat{a}(p) \equiv \frac{\pi y n(1-\sigma)}{(1-\pi)\left(M-\sigma^{2} m\right) p} .
$$

PROOF. In the Appendix.

The intuition behind Lemma 2 is as follows. The entrepreneurs who can restructure under both relationship and transactional funding $(a<\bar{a}(p, M))$ and those who cannot restructure under either type of

\footnotetext{
${ }^{6}$ We do not impose a limited liability constraint for the lender. Implicitly, we are assuming that the lender has more than enough funds to operate transfers to the entrepreneur on date 3, if needed. Adding a limited liability constraint would not alter the results.
} 
funding $(a \geq \bar{a}(p, m))$ choose relationship funding. In fact, for given innovation opportunities, a relationship lender offers cheaper credit because she is more efficient in liquidating mature assets. The entrepreneurs with $a \in[\bar{a}(p, M), \bar{a}(p, m)]$ face instead a trade-off: on the one hand, only transactional funding allows them to restructure; on the other hand, relationship funding offers them cheaper credit. The extra cost of transactional funding increases with the amount of collateral $a$ and equals the expected cost of the technological inertia induced by relationship funding when $a=\widehat{a}(p)$.

Thus far, we have focused on the entrepreneurs who obtain credit on date 1. In Lemma 3, we establish the conditions under which, for a given type of funding, an entrepreneur has indeed access to the credit market. Henceforth, we assume that a lender breaks a tie in favour of granting credit.

LEMMA 3: If it is anticipated that the lender will carry out the action necessary for the innovation, an entrepreneur will have access to credit if and only if $a \geq \underline{a}(p, \mu, A)$, where

$$
\underline{a}(p, \mu, A) \equiv \frac{i-\pi l y(1+n-n \sigma)}{\sigma^{2} p(1-\pi) \mu} .
$$

If, instead, it is anticipated that the lender will not carry out the action necessary for the innovation, an entrepreneur will have access to credit if and only if $a \geq \underline{a}(p, \mu, \bar{A})$, where

$$
\underline{a}(p, \mu, \bar{A}) \equiv \frac{i-\pi l y}{(1-\pi) \mu p} .
$$

PROOF. In the Appendix.

The intuition behind Lemma 3 is straightforward. The limited verifiability of their output and the low value of their collateral imply that some entrepreneurs may be unable to pledge enough returns to a lender. This can lead to the exclusion of these collateral-poor entrepreneurs from the credit market.

Together, Lemmas 1 and 3 illustrate the dual role of collateral and credit relationships in our model economy. Lemma 3 shows that they both ease entrepreneurs' access to credit: indeed, some entrepreneurs suffer from a shortage of collateral and are excluded from the credit market. Lemma 1 shows instead that an excess of collateral and credit relationships may inhibit entrepreneurs' restructuring activity: relationship lenders acquire high quality information on existing collateral and they can impede restructuring to preserve the value of their information.

From now on, we assume that, regardless of $\mu \in\{M, m\}$ and $\alpha \in\{A, \bar{A}\}$, borrowers with small enough collateral $a$ do not obtain credit. Precisely, we let $\inf _{\mu, \alpha} \underline{a}(p, \mu, \alpha)>0$, which implies

$$
\frac{i}{\pi l y}>1+n-n \sigma
$$

\subsection{The Asset Price}

In the previous section, we have solved for agents' decisions taking the market price of assets $p$ as given. We now solve for the asset price in general equilibrium. Denote by $\underline{a}$ the threshold value of collateral below 


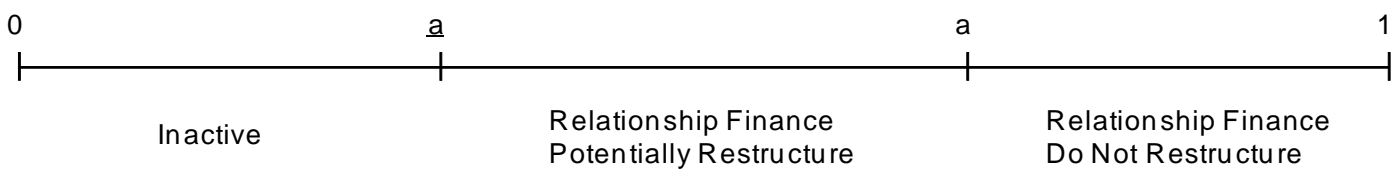

MIXED FINANCE REGIME (a<ã)

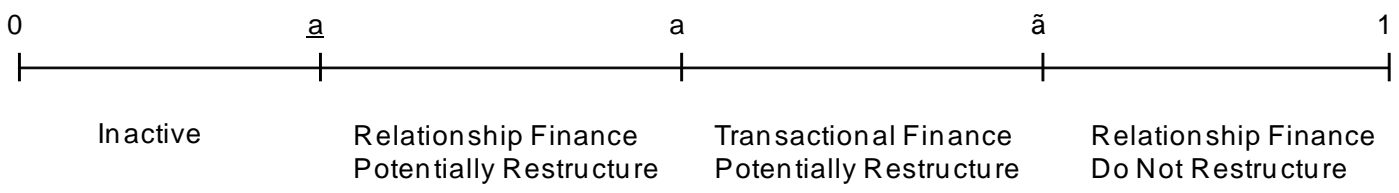

Figure 2: Firm Distribution Across Collateral Values.

which an entrepreneur cannot obtain credit. The demand $D$ of liquidated assets satisfies

$$
D(p)=\max \left[(1-\underline{a}) \pi\left(1-\frac{p}{\eta \bar{\theta}}\right), 0\right] .
$$

Intuitively, a measure $1-\underline{a}$ of entrepreneurs obtain credit and become active. Moreover, a share $\pi$ of active entrepreneurs is successful and remains in business. Finally, a share $(\eta \bar{\theta}-p) / \eta \bar{\theta}$ of the entrepreneurs who remain in business recover an output no lower than $p$ from reusing assets.

The supply $S$ of assets satisfies

$$
S(p)=\max \left\{(1-\pi) \int_{\underline{a}}^{1} a d a, 0\right\} .
$$

The supply is given by the probability $1-\pi$ that an entrepreneur fails times the amount of assets $a$ that are liquidated by a failed entrepreneur, integrated across active entrepreneurs. At the risk of belaboring this point, we remind the reader that in this case with $\phi=1$ the only threshold that matters for the determination of the asset supply is $\underline{a}$ while the distribution of firms between the new and the mature technology plays no role. An equilibrium where neither demand nor supply is rationed obtains when $D(p)=S(p){ }^{7}$

\subsection{Restrictions on the Parameters}

We are now in a position to solve jointly for firms' distribution across collateral values and for the asset price. A complete characterization of the equilibrium for all the parameter values consistent with (6) is provided in the Supplement. Here, we impose some restrictions on the parameters that allow us to focus on the scenario of interest. Broadly speaking, we are interested in a scenario in which we can investigate the

\footnotetext{
${ }^{7}$ In Appendix A, we investigate the properties of the excess collateral demand $D(p)-S(p)$.
} 
impact of a negative shock on firms' restructuring activity. Thus, we impose restrictions on the parameters that ensure that restructuring is desirable for firms and is also feasible for some of them, while for other firms it is hindered by lenders' technological inertia. In Section 5.2, we will evaluate the robustness of the analysis to relaxing these restrictions.

\subsubsection{Restructuring is feasible for some firms}

First, we restrict attention to cases where some restructuring is feasible. In order to guarantee that some restructuring activity is feasible, we need to assume that $\underline{a}(p, \mu, A)<\bar{a}(p, \mu)$. Substituting for the values of $\underline{a}(p, \mu, A)$ and $\bar{a}(p, \mu)$, the region of parameters that satisfy this condition is given by

$$
\frac{i}{\pi l y}<1+\frac{n}{1+\sigma} .
$$

We also restrict attention to cases where, if the restructuring of a firm can only be induced under transactional funding $(a \in[\bar{a}(p, M), \bar{a}(p, m)))$, it is not prevented by the firm's lack of access to the credit market $(a \geq \underline{a}(p, m, A))$. This implies that, if transactional funding is not chosen, it is because it is not optimal for the firm to do so, and not due to lack of credit. This condition requires $\underline{a}(p, m, A) \leq \bar{a}(p, M))$, that is,

$$
\frac{i}{\pi l y} \leq 1+n-\frac{n \sigma M}{M+\sigma m}
$$

Note that (9) is implied by (10).

\subsubsection{Restructuring is not feasible for all firms}

Next, we introduce a restriction on the parameters that implies that restructuring is not feasible for every firm.

$$
\bar{\theta}>\frac{1}{\eta} \frac{\frac{2 \pi[\pi l y(1+n)-i]^{2}}{\sigma[(1-\pi) m]^{2}}}{\frac{(3 \pi-1)[\pi l y(1+n)-i]}{(1-\pi) m}-\frac{i-\pi l y(1+n-n \sigma)}{\sigma M}} .
$$

Restriction (11) guarantees that the collateral asset price is not too low, otherwise for a lender the loss of collateral liquidation value induced by the restructuring of a firm would be negligible (and the lender would have no reason to prevent the firm's restructuring).

\subsubsection{Restructuring is desirable for firms}

We assume that, if the amount of collateralizable assets is relatively small and restructuring can be induced under relationship funding $(a \in[\underline{a}(p, M, A), \bar{a}(p, M)))$, it achieves the highest surplus. In other words, the direct benefit of the new technology (given by the productivity edge $n$ ) outweighs the indirect benefit of the mature technology (given by the difference $\left(1-\sigma^{2}\right) M p a$ in the liquidation value of collateral). To identify the parameter region where the latter condition is satisfied, observe that under relationship funding restructuring achieves a higher surplus than operating the mature technology if and only if

$$
(1-\sigma) \pi(1+n) y+\sigma[\pi y+(1 \overline{12} \pi) \sigma M p a]>\pi y+(1-\pi) M p a
$$


that is,

$$
a<a^{*}(p, M) \equiv \frac{\pi n y}{(1+\sigma)(1-\pi) M p} .
$$

Thus, we need $\bar{a}(p, M) \leq a^{*}(p, M)$, i.e.,

$$
\frac{i}{\pi l y} \geq 1+n-\frac{n \sigma}{l(1+\sigma)}
$$

In order to ensure that there is a non-empty region that satisfies (6), (9), (10), and (14), we need to assume that the output pledgeability $l$ is neither too small nor too large, taking values in the interval $l \in\left[\frac{1}{1+\sigma},(1-\sigma) \frac{M+\sigma m}{M-\sigma^{2} m}\right] .^{8}$ All in all, this implies that the parameter region of interest is given by

$$
\frac{i}{\pi l y} \in\left[1+n-\frac{n \sigma}{l(1+\sigma)}, 1+n-\frac{n \sigma M}{M+\sigma m}\right]
$$

\subsection{Firm Distribution}

We can partition the region of parameters defined by (15) into two credit regimes, that we label "relationship finance" regime and "mixed finance" regime. The relationship finance regime is characterized by an empty region of collateralizable assets where it is optimal for an entrepreneur to choose transactional funding $(\widehat{a}(p)<$ $\bar{a}(p, M) ;{ }^{9}$ the mixed finance regime is characterized by a non-empty region of collateralizable assets where it is optimal for an entrepreneur to choose transactional funding $(\widehat{a}(p) \geq \bar{a}(p, M))$. Note that $\widehat{a}(p)<\bar{a}(p, M)$ if and only if

$$
\frac{i}{\pi l y}<1+n-\frac{n \sigma M(1-\sigma)}{l\left(M-\sigma^{2} m\right)}
$$

Simple algebra shows that, given $l \in\left[(1-\sigma) \frac{1}{1-\sigma^{2}},(1-\sigma) \frac{M+\sigma m}{M-\sigma^{2} m}\right]$, the relationship finance regime and the mixed finance regime can both occur in the region of parameters given by (15). Relationship finance occurs in the region

$$
\frac{i}{\pi l y} \in\left[1+n-\frac{n \sigma}{l(1+\sigma)}, 1+n-\frac{n \sigma M(1-\sigma)}{l\left(M-\sigma^{2} m\right)}\right)
$$

and mixed finance occurs in the region

$$
\frac{i}{\pi l y} \in\left[1+n-\frac{n \sigma M(1-\sigma)}{l\left(M-\sigma^{2} m\right)}, 1+n-\frac{n \sigma M}{M+\sigma m}\right] .
$$

Proposition 1 characterizes the behavior of entrepreneurs and lenders in the region of parameters given by $(15)$.

PROPOSITION 1: (i) Regardless of the credit regime, a firm does not obtain credit if $a \leq \underline{a}(p, M, A)$;

\footnotetext{
${ }^{8}$ Note that this interval is not empty if and only if $\frac{M-m}{m}<\frac{1-\sigma^{2}}{\sigma}$.

${ }^{9}$ Note that, since transactional funding involves a lower surplus than relationship funding, the only reason to choose transactional funding is if it is required to induce restructuring, i.e., if $a \in[\bar{a}(p, M), \bar{a}(p, m))$. Now, we have already assumed that there is always credit available in the region where restructuring is only possible under transactional funding. Thus, if there is no transactional funding, it must be because it is never optimal ${ }_{3}$ i.e., $\widehat{a}(p)<\bar{a}(p, M)$.
} 
TABLE II.

Effects of $1 \%$ Collateral Shock

\begin{tabular}{|c|c|c|c|c|c|c|}
\hline $\begin{array}{l}\text { Parameters } \\
\text { (Technology) }\end{array}$ & $\begin{array}{c}\text { Parameters } \\
(\text { Credit Mkt }) \\
\end{array}$ & Thresholds & & $\begin{array}{c}\text { Effects } \\
(R \text { eal Secto } \\
\end{array}$ & & $\begin{array}{c}\text { Effects } \\
\text { (Credit Market) } \\
\end{array}$ \\
\hline \multirow{6}{*}{$\begin{array}{l}\pi=0.94 \\
y=0.96 \\
n=0.09 \\
i=0.88 \\
l=0.93\end{array}$} & \multicolumn{6}{|c|}{ (Panel A: Flexible Credit Regime) } \\
\hline & $M=0.90$ & $\underline{a}(p, M, A)=0.7425$ & $\frac{\Delta I}{I}$ & $-2.9889 \%$ & \multirow{2}{*}{$\begin{array}{l}\frac{\Delta C R}{C R} \\
\frac{\Delta C}{C}\end{array}$} & $-2.9889 \%$ \\
\hline & $m=0.88$ & $\bar{a}(p, M)=0.8047$ & $\frac{\Delta R F}{R F}$ & $+1.0364 \%$ & & $-2.9889 \%$ \\
\hline & & $p=1$ & & $-1.0257 \%$ & & \\
\hline & & & $\frac{\Delta Y}{Y}$ & & & \\
\hline & & & & & & \\
\hline$\sigma=0.8$ & \multicolumn{6}{|c|}{ (Panel B: Conservative Credit Regime) } \\
\hline$\eta=1$ & $M=0.99$ & $\underline{a}(p, M, A)=0.6735$ & $\frac{\Delta I}{I}$ & $-2.1321 \%$ & $\frac{\triangle C R}{C R}$ & -2.2060 \\
\hline $\bar{\theta}=1.06$ & $m=0.98$ & $\bar{a}(p, M)=0.7298$ & $\frac{\Delta R F}{R F}$ & $+1.0338 \%$ & $\frac{\Delta C}{C}$ & -2.1321 \\
\hline$\phi=1$ & & $\widetilde{a}(p)=0.7373$ & $\underline{\Delta p}$ & $-1.0232 \%$ & & \\
\hline & & $p=1.0023$ & $\frac{\Delta Y}{Y}$ & & & \\
\hline
\end{tabular}

Note. The table reports a parameter selection (first and second column), implied collateral thresholds (third column), and the effects of a $1 \%$ drop in collateral productivity.

(ii) The outcome is $(M, A)$, that is, a firm chooses relationship funding and potentially restructures, if $a \in(\underline{a}(p, M, A), \bar{a}(p, M))$;

(iii) If $a \geq \bar{a}(p, M)$, then (a) in the region of parameters where the relationship finance regime occurs, the outcome is $(M, \bar{A})$, that is, a firm chooses relationship funding and does not restructure; (b) in the region of parameters where the mixed finance regime occurs, the outcome is $(m, A)$, that is, a firm chooses transactional funding and restructures, if $a \in[\bar{a}(p, M), \min \{\bar{a}(p, m), \widehat{a}(p)\})$; the outcome is instead $(M, \bar{A})$, that is, a firm chooses relationship funding and does not restructure, if $a \geq \min \{\bar{a}(p, m), \widehat{a}(p)\}$;

(iv) Regardless of the credit regime, the asset price satisfies

$$
p^{*}=\frac{\eta \bar{\theta}}{2 \pi} \frac{3 \pi-1}{2}+\left[\left(\frac{\eta \bar{\theta}}{2 \pi} \frac{3 \pi-1}{2}\right)^{2}-\frac{\eta \bar{\theta}}{2 \pi} \frac{i-\pi l y(1+n-n \sigma)}{\sigma^{2} M}\right]^{\frac{1}{2}} .
$$

PROOF. In the Appendix.

Figure 2 illustrates the two credit regimes. The relationship finance regime in (ii) arises when lenders are not prone to induce technological inertia within credit relationships, that is, relationship lenders prevent only firms with large collateral from restructuring their activity. Alternatively, this credit regime arises when entrepreneurs derive a large benefit from credit relationships, that is, they obtain much cheaper financing from relationship lenders. In this credit regime, all firms form credit relationships and firms only differ in their technology choice: those with medium collateral potentially restructure whereas those with large collateral adopt the mature technology. The mixed finance regime in (iii) arises when lenders are prone to induce technological inertia within credit relationships or entrepreneurs derive a small benefit from relationships. In this regime, firms with medium collateral value avoid the technological inertia induced by relationship lenders by choosing transactional funding. As in the relationship finance regime, firms with medium collateral value potentially restructure while firms with large collateral adopt the mature technology. In sum, the critical 
difference between the two credit regimes consists of the role of transactional finance. In the relationship finance regime, transactional finance is inactive; in the mixed finance regime, it is active and specializes in the financing of firms that potentially restructure their activity. In both regimes, collateral-rich firms form credit relationships with lenders in which they preserve the mature technology.

While Proposition 1 derives a closed form solution, to fix ideas it is useful to consider two numerical examples. Consider the parameters in Table II, first column and Panel A (second column). These parameters imply that the probability $1-\pi$ of failure of a project is $6 \%$; when the innovation occurs, the return from a project $y(1+n) / i-1$ in the event of success net of the investment cost equals $19 \%$; in the event of success, the return of the new technology exceeds that of the mature by $9 \%(=n)$; if the lender carries out the action, the probability $1-\sigma$ of innovation is $20 \% ; 7 \%(1-l)$ of the output is non-verifiable; finally, the share of value lost in liquidation by a relationship (transactional) lender amounts to $1-M=10 \%(1-m=12 \%)$. With this parameter selection the economy is in the relationship finance regime. Consider next the parameters in Table II, first column and Panel B (second column). These parameters are the same as before, except that the share of value lost in liquidation by a relationship (transactional) lender amounts to $1 \%(2 \%)$. With this parameter selection the economy is in the mixed finance regime.

In the two numerical examples, we have kept the values of the technological parameters fixed and we have allowed only the parameters of the credit market $M$ and $m$ to vary. The purpose of this exercise is to disentangle the role of lenders' information. Using the parameterization in Table II, it is possible to show that, for a given level of $M$, a wider gap between $M$ and $m$ pushes the economy into the relationship finance regime. In fact, for a given degree of technological inertia, a wider gap $M-m$ implies a larger benefit from credit relationships (i.e., a larger gap between the cost of transactional finance and the cost of relationship finance). In turn, for a given gap $M-m$, a higher $M$ pushes the economy into the mixed finance regime (where transactional finance is active). In fact, for a given benefit of credit relationships, a higher value of $M$ exacerbates the technological inertia of relationship lenders. All in all, this implies that in the $(M, M-m)$ space the frontier between the two credit regimes is upward sloping.

\section{Impact of Shocks}

We now study the effects of shocks in the relationship finance regime and in the mixed finance regime. We are primarily interested in the effects of a shock to collateral that is akin to the collateral squeeze considered in Holmstrom and Tirole (1997) (see also Gertler and Karadi, 2010, for example). We let this shock take the form of a drop in the aggregate productivity $\eta$ of liquidated assets and assume that the drop in $\eta$ is small so we can evaluate its effects with the help of differential calculus. We also investigate the effects of a shock to productivity that takes the form of a drop in $y$. In practice, as in Holmstrom and Tirole (1997), for example, we perform comparative statics exercises, comparing the equilibrium that obtains in our economy for two different values of $\eta$ (in the case of a collateral shock) and for two different values of $y$ (in the case of 
a productivity shock). For instance, when we say that a credit relationship breaks down after the collateral shock, we mean that for the higher value of $\eta$ the firm would have borrowed from a relationship lender, while for the lower value of $\eta$ it borrows from a transactional lender or it is inactive. In the real sector, we focus on the effects on total investment $(I)$, on the measure of restructuring firms $(R F)$, on output $(Y)$ and on the asset price $\left(p^{*}\right)$. In the credit sector, we focus on the effects on the measure of firms that have access to the credit market $(C)$ and on the measure of firms that engage in credit relationships $(R)$.

Proposition 2 summarizes the effects of the collateral shock in the two credit regimes.

PROPOSITION 2: In both the relationship finance regime and the mixed finance regime, a negative collateral shock (reduction of $\eta$ ) reduces the asset price, total investment, the measure of firms that have access to credit, and the measure of firms that engage in credit relationships. By contrary, a negative collateral shock increases the measure of restructuring firms. The effect on output is ambiguous: the decrease in the measure of firm that have access to credit reduces output while the increase in the measure of restructuring firms increases output.

PROOF. In the Appendix.

The drop in the productivity $\eta$ of assets induces a fall of the asset price $p^{*}$ because, for a given price, the demand for liquidated assets shrinks. The measure of firms that have access to the credit market falls with $p^{*}$. In particular, the firms that were "marginal" in the credit market (that is, with initial collateral in the neighborhood of $\left.\underline{a}\left(p^{*}, M, A\right)\right)$ are denied credit because they can no longer pledge enough expected returns to a lender. These firms drop out of the credit market, their investment is lost and their credit relationships break down. Clearly, the exclusion of these firms from the credit market further reduces the demand and the supply of assets and feeds back on the asset price. This effect of the collateral shock on collateral-poor firms is similar to that obtained by Holmstrom and Tirole (1997).

The new prediction of the model is the impact of the shock on collateral-rich firms. Consider first the relationship finance regime. Since the threshold collateral $\bar{a}\left(p^{*}, M\right)$ above which collateral-rich firms face technological inertia within credit relationships is positively related to the asset price $p^{*}$, the shock allows firms in the neighborhood of $\bar{a}\left(p^{*}, M\right)$ to potentially restructure their activity within their credit relationships. Next, consider the mixed finance regime. Firms with initial collateral in the neighborhood of $\min \{\bar{a}(p, m), \widehat{a}(p)\}$ prefer now borrowing from transactional lenders and restructuring their activity (in fact, $\min \{\bar{a}(p, m), \widehat{a}(p)\}$ is negatively related to $\left.p^{*}\right)$. These firms deliberately break their credit relationships, borrow from new investors, and restructure. In both credit regimes, the assumption of a uniform distribution of firms' collateral values implies that the measure of additional collateral-rich firms that potentially restructure their activity outweighs the measure of firms squeezed out by the shock from the credit market. Hence, the measure of restructuring firms increases. ${ }^{10}$ It is also worth observing that in general equilibrium the

\footnotetext{
${ }^{10}$ With a generic distribution, whether the measure of restructuring firms increases or decreases depends on the measure of this group of firms relative to those squeezed out by the shock from the credit market. Of course, there are sufficiently right-
} 
exclusion of collateral-poor firms from the credit market is beneficial for the restructuring of collateral-rich firms. In fact, when collateral-poor firms become inactive, the net demand for collateral assets drops. This further depresses the collateral asset price, and, given the negative relationship between $\bar{a}\left(p^{*}, M\right)$ and $p^{*}$ (in the relationship finance regime) and between $\min \{\bar{a}(p, m), \widehat{a}(p)\}$ and $p^{*}$ (in the mixed finance regime), it fosters the restructuring of collateral-rich firms. This will be especially relevant for evaluating the impact of policies that sustain the access of collateral-poor firms to the credit market.

Turning to the impact of the shock on output, in the relationship finance regime this reflects the interaction between two opposite forces. On the one hand, the loss of investment due to the exclusion of collateral-poor firms from the credit market tends to decrease output. On the other hand, the increase of restructuring of collateral-rich firms tends to increase output. In the mixed finance regime, if the asset liquidation costs are real, there is also a third additional force which tends to depress output. To understand this third mechanism, however, we need to investigate the effects of the shock in the credit market.

Proposition 2 implies that the qualitative effects of the collateral shock on restructuring activity and investment are the same in the two credit regimes. What differs is the way firms' restructuring occurs in the credit market. In the relationship finance regime, the restructuring of collateral-rich firms occurs within their credit relationships and the breakdown of relationships caused by the shock is entirely attributable to the exclusion of collateral-poor firms from the credit market. In the mixed finance regime, instead, the restructuring of collateral-rich firms entails the breakdown of their credit relationships, so that the surge in firms' restructuring thus leads to an additional breakdown of credit relationships besides that induced by the exclusion of collateral-poor firms from the credit market. This additional breakdown is only partially compensated by the fact that in the mixed finance regime firms with initial collateral in the neighborhood of $\bar{a}(p, M)$ are now allowed to restructure by relationship lenders and prefer relationship funding. These arguments are formalized in Proposition 3.

PROPOSITION 3: In the relationship finance regime, a negative collateral shock (reduction of $\eta$ ) induces the same percentage drop in the measure of active firms and in the measure of credit relationships. In the mixed finance regime, the percentage drop in the measure of credit relationships is larger than the percentage drop in the measure of active firms. Precisely, the change in the ratio of credit relationships over active firms is given by

$$
\frac{\partial\left(\frac{R_{m}}{C}\right)}{\partial \eta}=\frac{C-R_{m}}{C^{2}} \frac{1}{p^{*}} \frac{\partial p^{*}}{\partial \eta}>0
$$

PROOF. In the Appendix.

It should now be clear why in the mixed finance regime there is a third additional force which tends

skewed distributions such that the measure of restructuring firms drops. However, even in this case, the ratio "(restructuring firms)/(total firms)" may increase. Finally, if this ratio declines too, the predictions of the model will resemble those of the studies on the negative effects of credit imperfections on restructuring activity during recessions (e.g., Caballero and Hammour, 2004; Barlevy, 2003). 
to depress output after the collateral shock. When the liquidation costs are at least partially real, the additional breakdown of credit relationships that is induced by firms' restructuring activity implies a surge in liquidation costs. In other words, in the mixed finance regime the output change is the result of three competing forces. Besides the output drop due the decline of investment and the output increase due to the increased restructuring, there is also the increase in transaction costs due to the voluntary breakdown of credit relationships by collateral-rich firms. The latter effect does not occur in the relationship finance regime.

The effects of the shock can be further grasped with the help of the two numerical examples in Table II. Consider again the parameters in Table II, first column and Panel A (second column). A reduction of $\eta$ by $1 \%$ triggers $\Delta C R / C R=\Delta I / I=\Delta C / C \cong-2.9889 \%, \Delta R F / R F \cong+1.0364 \%, \Delta p / p \cong-1.0257 \%$. Consider next the parameters in Table II, first column and Panel B (second column). Then, $\Delta C R / C R=$ $-2.2060>\Delta C / C=\Delta I / I \cong-2.1321 \%, \Delta R F / R F \cong+1.0338 \%, \Delta p / p \cong-1.0232 \%$.

As anticipated, while our primary focus is on collateral shocks, we are also interested in studying the effects of a productivity shock, that is a reduction in $y$. As above, we assume that the shock is sufficiently

small so we can evaluate its effects with the help of differential calculus. Proposition 4 summarizes the results.

PROPOSITION 4: In both the relationship finance regime and the mixed finance regime, a negative productivity shock reduces the asset price, total investment, the measure of firms that have access to credit, and the measure of firms that engage in credit relationships. A negative productivity shock also reduces the measure of restructuring firms and output. However, the drop in the measure of restructuring firms is smaller than the drop in total investment.

PROOF. In the Appendix.

Given what said about collateral shocks, the intuition behind Proposition 4 is straightforward. The direct negative effect of a drop in productivity, which especially hits the return of the new technology, is partially dampened by the fact that, at any productivity $y$, a decrease in the price level increases the restructuring of collateral-rich firms. Thus, the drop in restructuring is smaller than the drop in investment.

\section{Robustness}

This section explores the robustness of the analysis. We first relax the assumption that mature and new assets have the same redeployability in the asset market. We then illustrate what would happen in credit regimes alternative to the two regimes considered in the previous section. 


\subsection{Asset Heterogeneity}

In the baseline analysis, we assumed that mature and new assets have the same degree of redeployability in the asset market $(\phi=1)$. This implies that the supply of assets does not depend on the distribution of firms between the new and the mature technology, but only on the measure of firms that have access to the credit market. Hence, when a negative collateral shock hits, there is a contraction in the demand and in the supply of assets due to the dropout of some firms from the credit market. However, the change in the measure of restructuring firms has no feedback effect on the asset price. By contrary, in a context in which the redeployability of the assets of the new technology is lower than the redeployability of the assets of the mature technology $(\phi<1)$, the increase in the measure of restructuring firms shrinks the supply of assets because less assets will be resold in the market. For example, in the relationship finance regime the asset supply equals

$$
S(p)=\max \left\{(1-\pi)\left[\int_{\underline{a}(p, M, A)}^{\bar{a}(p, M)}[(1-\sigma) \phi+\sigma] a d a+\int_{\bar{a}(p, M)}^{1} a d a\right], 0\right\} .
$$

In turn, the reduction in the supply of assets due to the increased restructuring tends to sustain the asset price dampening both the increase of restructuring activity and the decline of investment. In Proposition 5, we show that in this alternative case with $\phi<1$ the qualitative results of Proposition 1 remain unchanged, although the effects of the shock are dampened.

PROPOSITION 5: Suppose $\phi<1$. Both in the relationship finance regime and in the mixed finance regime, the negative collateral shock has the same effects as those illustrated in Proposition 2. However, all the effects of the shock, including the increase in firms' restructuring activity, are smaller.

PROOF. In the Appendix.

An interesting interpretation of this result is that if the innovation is "radical", so that the assets of the new technology significantly differ from the assets of the existing technology and, hence, are less easily redeployable in the market, the increase in restructuring activity induced by a collateral shock can be less important. At the same time, however, Proposition 5 suggests that the drops in investment, asset price and output can also be smaller than in the case of an "incremental" innovation with assets similar to those of the existing technology.

\subsection{Other Credit Regimes}

In the baseline analysis, we introduced restrictions on the parameters that allowed us to focus on two credit regimes of interest. We now briefly discuss what happens in the complementary region of the parameter space. A complete characterization of the equilibrium for all the parameter values consistent with (6) is provided in the Supplement. 
First, we considered only cases where some restructuring activity is feasible. Clearly, if we impose that restructuring is never feasible, ${ }^{11}$ the only equilibrium outcome in the presence of credit is $(M, \bar{A})$, i.e., relationship funding and no restructuring. In this scenario, a negative collateral shock would only have the standard effect of a contraction in aggregate investment due to tightening collateral constraints. We also restricted attention to cases where, whenever transactional funding is required for restructuring (i.e., $a \in[\bar{a}(p, M), \bar{a}(p, m)))$, it is not prevented by lack of credit. If we drop this restriction, two scenarios can arise. First, it may be that, in a subset of $[\bar{a}(p, M), \bar{a}(p, m))$, the outcome is $(M, \bar{A})$ because credit is only available under relationship funding. Second, it may be that the outcome is $(M, \bar{A})$ in a subset of $[\bar{a}(p, M), \bar{a}(p, m))$ because, even though transactional funding is feasible, it is not desirable for firms. In both scenarios, the key implication is that now there is a threshold value of collateral $a^{\circ}(p) \in[\bar{a}(p, M), \bar{a}(p, m))$ such that the equilibrium outcome is $(m, A)$ for all $a \in\left[\bar{a}(p, M), a^{\circ}(p)\right)$, and the equilibrium outcome is $(M, \bar{A})$ for all $a \in\left[a^{\circ}(p), \bar{a}(p, m)\right)$. Broadly speaking, such a distribution of firms across collateral values is not very different from the distribution in a mixed finance regime.

In the baseline analysis, we also assumed that there exists an upper tail in the distribution of collateral values in which the technological inertia of lenders arises (i.e., restructuring is not feasible for all firms). In fact, we considered a region of parameters (basically, a value of $\bar{\theta}$ sufficiently large) such that this indeed happens. Whether this assumption is realistic or not - and how fat this upper tail of the distribution of collateral assets is - depends on the structural characteristics of the economy. In an economy in which very few firms have large collateral, or where new technologies have a significant productivity advantage over mature ones, this condition will not hold and the equilibrium outcome in the presence of credit will always be $(M, A)$. In this case, the only mechanism at work during a credit crunch will be the traditional mechanism of contraction of investment and of restructuring activity due to tightening collateral constraints.

Finally, in the baseline analysis we assumed that, if restructuring under relationship funding is feasible, it achieves the highest surplus. If we relax this assumption, the key implication is that now there will exist a subset of $a \in[\underline{a}(p, M, A), \bar{a}(p, M))$ where, even though the outcome $(M, A)$ is feasible, it is not desirable for firms and the entrepreneurs will prefer relationship funding and no restructuring. Broadly speaking, this scenario is not very different from the distribution in the relationship finance regime.

\section{Extensions: Credit and Asset Market Policy}

During the 2008-2010 crisis, the Federal Reserve engaged in two kinds of unconventional policy (Krishnamurty, 2010). First, it intervened directly in asset markets to sustain declining asset prices (for instance, by purchasing mortgage backed securities). Second, it directly granted loans to firms and non-bank financial institutions to finance asset holdings at margin requirements lower than those applied by private financial

\footnotetext{
${ }^{11}$ This occurs, for instance, if the output verifiability is very low so that for collateral-poor firms it is actually the mature technology that can offer more pledgeable expected returns to a lender.
} 
institutions. The model can help understand the consequences of these two policies for firms' restructuring activity, besides their consequences for total investment. We are going to see that an unappealing consequence of these policies could be that while, as intended, they support investment, they also tend to freeze the increase in firms' restructuring activity triggered by a credit crunch.

Throughout this section, we work with the baseline scenario in which $\phi=1$. For conciseness, we also restrict attention to the relationship finance regime and assume that all the transaction costs sustained for asset liquidation are nominal (the qualitative insights carry through to the mixed finance regime and to the case of real transaction costs). Following Krishnamurty (2010), we can think of the first policy (intervention in the asset market) as consisting of the government subsidizing asset purchases in the liquidation market on date 3. Precisely, we posit that the government makes a transfer $\tau$ of final good to each entrepreneur who purchases one unit of liquidated assets. We have also to specify how the government finances these subsidies for asset purchases. In our economy, the government can levy non-distortionary (lump-sum) taxes. For example, on date 3 it may tax the revenues of investors, regardless of whether these revenues originate from storage, from the repayment of loans or from asset liquidation; alternatively, it may tax the output of collateral-rich entrepreneurs without incurring the risk that such taxation distorts agents' decisions.

By sustaining the asset demand, the subsidy raises the asset price. This increases the access of collateralpoor firms to credit but reduces the collateral threshold $\bar{a}\left(p^{*}(\tau), M\right)$ above which entrepreneurs are prevented from adopting the new technology by their relationship lenders. The latter effect generates an expected output loss of $(1-\sigma) \pi y n$ per project (the expected output advantage of the new technology times the probability of the technological innovation). We obtain that the change in output due to the transfer is

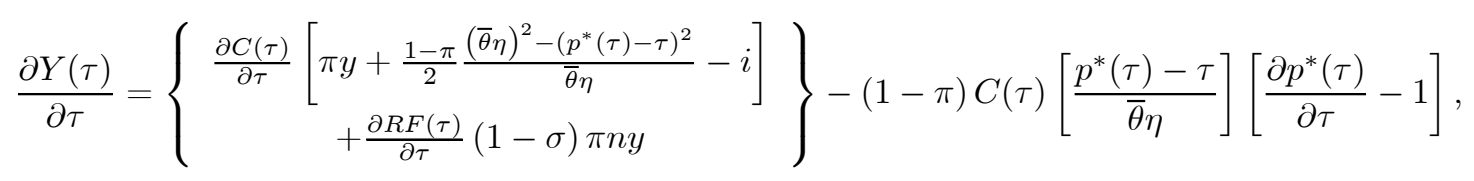

where

$$
\frac{\partial C(\tau)}{\partial \tau}=\frac{i-\pi l y(1+n-n \sigma)}{\sigma^{2}(1-\pi) M\left[p^{*}(\tau)\right]^{2}} \frac{\partial p^{*}(\tau)}{\partial \tau}>0
$$

and

$$
\frac{\partial R F(\tau)}{\partial \tau}=-\frac{\pi l y(1+n+\sigma)-i(1+\sigma)}{\sigma^{2}(1-\pi) M\left[p^{*}(\tau)\right]^{2}} \frac{\partial p^{*}(\tau)}{\partial \tau}<0
$$

are respectively the increase in the measure of firms that have access to credit and the decrease in the measure of firms that restructure their activity. Intuitively, an increase in $\tau$ may lead to a decrease in output as long as $\left|\frac{\partial R F(\tau)}{\partial \tau}\right|$ is sufficiently larger than $\frac{\partial C(\tau)}{\partial \tau}$. This occurs, for instance, if $i$ is close enough to $\pi l y(1+n-n \sigma)$. In this case, $\frac{\partial p^{*}(\tau)}{\partial \tau} \approx 1, \frac{\partial C(\tau)}{\partial \tau} \approx 0$ and

$$
\frac{\partial R F(\tau)}{\partial \tau} \approx-\frac{\pi l y n}{(1-\pi) M\left[\frac{\eta \bar{\theta}}{\pi}\left(\frac{3 \pi-1}{2}+\frac{\pi}{\eta \bar{\theta}} \tau\right)\right]^{2}}<0
$$

The second policy consists of the government directly lending to firms in the credit market at margin requirements lower than private lenders. We model this policy assuming that the government grants credit 
to firms with $a \in\left[\underline{a}_{G}, \underline{a}(p, M, A)\right]$, where the policy tool is now the $\underline{a}_{G}$ threshold. Similar to the first policy, we posit that the government finances any loss due to this policy by levying lump-sum taxes. The change in output due to the direct lending policy is

$$
\frac{\partial Y\left(\underline{a}_{G}\right)}{\partial \underline{a}_{G}}=\left\{\begin{array}{c}
\frac{\partial C\left(\underline{a}_{G}\right)}{\partial \underline{a}_{G}}[ \\
{\left[\pi y+\frac{1-\pi}{2} \frac{(\bar{\theta} \eta)^{2}-\left(p^{*}\left(\underline{a}_{G}\right)\right)^{2}}{\bar{\theta} \eta}-i\right]} \\
+\frac{\partial R F\left(\underline{a}_{G}\right)}{\partial \underline{a}_{G}}(1-\sigma) \pi n y
\end{array}\right\}-(1-\pi) C\left(\underline{a}_{G}\right) \frac{p^{*}\left(\underline{a}_{G}\right)}{\bar{\theta} \eta} \frac{\partial p^{*}\left(\underline{a}_{G}\right)}{\partial \underline{a}_{G}} .
$$

Note that

$$
\frac{\partial Y(0)}{\partial \underline{a}_{G}}=-\left\{\begin{array}{c}
\pi y+(1-\sigma) \pi n y \\
+\bar{\theta} \eta \frac{1-\pi}{2}\left[1-\left(\frac{3 \pi-1}{2 \pi}\right)^{2}\right]-i
\end{array}\right\}+\frac{1-\pi}{3 \pi-1}\left[\bar{a}\left(p^{*}(0), M\right)(1-\sigma) \pi n y+(1-\pi) \bar{\theta} \eta\left(\frac{3 \pi-1}{2 \pi}\right)^{2}\right]
$$

is positive as long as

$$
(1-\sigma) \pi n y\left[\frac{2 \pi}{(3 \pi-1)^{2}} \frac{\pi l y(1+n)-i}{\sigma M \bar{\theta} \eta}-1\right]>\pi\left[y+\bar{\theta} \eta\left(\frac{1-\pi}{2 \pi}\right)^{3}\right]-i .
$$

For instance, this occurs when $n$ is large enough. In this case, the optimal policy does not involve offering credit to all firms.

To summarize, with both kinds of policy, the government intervention tends to freeze the increase in the restructuring of collateral-rich firms after the collateral shock. Since the technological inertia induced by relationship lenders is stronger when the asset price is higher, policies that aim at sustaining the asset price during a credit crunch foster total investment but dampen the increase in the restructuring of collateral-rich firms. The same effect occurs when the government directly provides funding to collateral-poor firms that would otherwise be denied credit after the credit crunch. In fact, the credit rationing of collateral-poor firms depresses the net demand of collateral assets, reducing the asset price and fostering the restructuring of collateral-rich firms. A policy of direct lending to collateral-poor firms dampens this general equilibrium effect.

For a given reduction in $\underline{a}(p, M, A)$, the government intervention tends to freeze the increase in restructuring less when a policy of direct lending to collateral-poor firms is implemented. Intuitively, sustaining the asset price by purchasing assets tends to directly depress firms' restructuring activity while the dampening effect induced by the policy of direct lending is only indirect. Since $C\left(\underline{a}_{G}\right)=1-\underline{a}_{G}$, in order to verify this formally, we need to show that

$$
\left.\frac{\partial p^{*}(\tau)}{\partial \tau}\right|_{\frac{\partial C(\tau)}{\partial \tau}=1}>-\frac{\partial p^{*}\left(\underline{a}_{G}\right)}{\partial \underline{a}_{G}} .
$$

After some manipulations, this can be rewritten as

$$
\left(\frac{3 \pi-1}{2}+\frac{\pi}{\eta \bar{\theta}} \tau\right)^{2}>\frac{2 \pi}{\eta \bar{\theta}} \frac{i-\pi l y(1+n-n \sigma)}{\sigma^{2} M} .
$$

This condition is trivially satisfied when $i$ is sufficiently close to $\pi l y(1+n-n \sigma)$, for instance. 


\section{Conclusion}

We have investigated the role of the credit market in an economy where firms can restructure their activity and switch to new technologies more productive than existing ones. In our economy, credit relationships ease information flows between entrepreneurs and lenders and, hence, entrepreneurs' access to the credit market. However, relationship lenders inhibit the restructuring of collateral-rich firms to preserve the value of their information on mature technologies. By depressing the price of collateral assets, a negative collateral shock squeezes collateral-poor firms out of the credit market but fosters the restructuring of collateral-rich firms. Depending on the credit regime that prevails in equilibrium, the restructuring of collateral-rich firms can occur with or without the breakdown of their credit relationships. In the last part of the analysis, we have also investigated the consequences of policies of direct government intervention in the credit and asset markets. We have found that the credit and asset market policies adopted during the recent credit crunch can promote investment but might also slow down a process of restructuring in the credit market.

The analysis leaves important questions open for future research. While the model can help disentangle the mechanisms through which a credit crunch affects technological change, it cannot offer predictions on the magnitude of the effects. A priority for future research is thus to cast the analysis into a dynamic general equilibrium environment and study the quantitative relevance of the mechanisms uncovered in this paper.

\section{APPENDIX}

\subsection{PROOF OF LEMMA 1}

The spread $r_{n}-r$ is maximum when $r_{n}$ is set at its upper limit. The maximum level of $r_{n}$ that can be set in the contract is $l y(1+n)$. Given this choice of $r_{n}$, the minimum level of $r$ that guarantees non-negative expected profits to the lender if the innovation can occur satisfies

$$
\pi r=\frac{i-\pi(1-\sigma) l y(1+n)}{\sigma}-(1-\pi) p \sigma \mu a .
$$

By plugging these values of $r_{n}$ and $r$ into the left hand side of (1), and operating algebraic manipulations, we obtain the condition $a<\bar{a}(p, \mu)$.

\subsection{PROOF OF LEMMA 2}

Here we focus on the formal part of the proof; the rest is in the main text. As we argue in the main text, the non-obvious case is when only a transactional lender allows the entrepreneur to restructure, i.e. $a \in[\bar{a}(p, M), \bar{a}(p, m))$. In this case, it is easy to see that the condition under which an entrepreneur chooses transactional funding is

$$
\pi\left[(1-\sigma)\left[y(1+n)-r_{n}\right]+\sigma(y-\widetilde{r})\right]>\pi(y-\widehat{r}) .
$$

The repayments $\widetilde{r}$ and $r_{n}$ that guarantee zero profits to a transactional lender satisfy

$$
\pi\left[(1-\sigma) r_{n}+\sigma \widetilde{r}\right]=i-(1-\pi) \sigma^{2} p m a,
$$


whereas the repayment $\widehat{r}$ that guarantees zero profits to a relationship lender satisfies

$$
\pi \widehat{r}=i-(1-\pi) M p a .
$$

Using (34) and (33) to substitute into (32), we obtain

$$
\widehat{a}(p) \equiv \frac{\pi y n(1-\sigma)}{(1-\pi)\left(M-\sigma^{2} m\right) p} .
$$

\subsection{PROOF OF LEMMA 3}

If the lender implements the action that allows the innovation, the entrepreneur obtains credit if and only if

$$
(1-\sigma) \pi l y(1+n)+\sigma[\pi l y+(1-\pi) \mu \sigma p a] \geq i .
$$

We can rewrite this inequality as

$$
a>\frac{i-\pi l y[1+n-n \sigma]}{(1-\pi) \mu \sigma^{2} p} .
$$

In turn, if the lender does not implement the action that allows innovation, the entrepreneur obtains credit if and only if

$$
\pi l y+(1-\pi) \mu p a \geq i
$$

that is,

$$
a \geq \frac{i-\pi l y}{(1-\pi) \mu p}
$$

\subsection{PROOF OF PROPOSITION 1}

First, note that $\underline{a}(p, M, A) \leq \underline{a}(p, M, \bar{A})$ if and only if $\frac{i}{\pi l y} \leq 1+n-n \sigma M \frac{1}{M+\sigma m}$, which is implied by (15). Thus, regardless of whether the lender implements or not the action, an entrepreneur with $a \leq \underline{a}(p, M, A)$ does not have access to credit. Now, let $a \in[\underline{a}(p, M, A), \bar{a}(p, M))$. In this case, the outcome $(M, A)$ is feasible and achieves the highest surplus. Thus, since the lender only receives a fixed payoff $i$ that compensates for her investment, the outcome $(M, A)$ must be chosen by the entrepreneur. Now, let $a \geq \bar{a}(p, M)$ and consider the relationship finance regime. In this regime, $\widehat{a}(p) \leq \bar{a}(p, M)$ and it is never optimal for the entrepreneur to choose transactional funding. Moreover, $a \geq \bar{a}(p, M)$ implies that the entrepreneur cannot choose a repayment scheme that induces the lender to implement the innovation. Thus, if credit is extended, the equilibrium outcome must be $(M, \bar{A})$. Now, note that $\underline{a}(p, M, \bar{A})<\bar{a}(p, M)$ if and only if $\frac{i}{\pi l y}<1+\frac{n}{1+\sigma}$, which is always true in the region of parameters given by (15). Finally, let $a \geq \bar{a}(p, M)$ and consider the mixed finance regime. There are two cases to be considered, depending on the relation between $\bar{a}(p, m)$ and $\widehat{a}(p)$. First, let us assume that $\bar{a}(p, m) \leq \widehat{a}(p)$. In this case, for all $a \geq \bar{a}(p, m)$, restructuring can never occur and the outcome must be $(M, \bar{A})$. We already know that this outcome can be funded in the region of parameters given by (15). In turn, for all $a \in[\bar{a}(p, M), \bar{a}(p, m))$, restructuring under transactional funding is feasible and optimal. Thus it must be chosen and the outcome is $(m, A)$. Next, let us assume that $\bar{a}(p, m)>\widehat{a}(p)$. In this case, for all $a \in[\bar{a}(p, M), \widehat{a}(p))$, the outcome $(m, A)$ is chosen since it is feasible and optimal. It remains to be considered the case $a \geq \widehat{a}(p)$. In this case, since restructuring and transactional funding is no longer an optimal outcome and restructuring cannot be accomplished with relationship funding, the outcome must be $(M, \bar{A})$. Again, we know that this outcome is feasible in the region of parameters given by (15). 
To complete our proof, we obtain the equilibrium asset price. Remember that firms' collateral $a$ is uniformly distributed in the interval $[0,1]$ and that a firm's ability to reuse assets is given by $\eta \theta$, where $\theta$ is uniformly distributed in the interval $[0, \bar{\theta}]$. This implies that the demand for assets is

$$
D(p)=\max \left\{[1-\underline{a}(p, M, A)] \pi\left(1-\frac{p}{\eta \bar{\theta}}\right), 0\right\},
$$

while the supply of assets is

$$
S(p)=\max \left\{(1-\pi) \int_{\underline{a}(p, M, A)}^{1} a d a, 0\right\}
$$

which can be rewritten as

$$
S(p)=\max \left\{\frac{1}{2}(1-\pi)\left[1-(\underline{a}(p, M, A))^{2}\right], 0\right\} .
$$

Clearly, if $p$ is such that $\underline{a}(p, M, A) \geq 1$, then both supply and demand are equal to zero. This is an uninteresting equilibrium because it implies that no firm receives credit. Thus, in what follows we consider equilibria where $\underline{a}(p, M, A)<1$, that is,

$$
p>\frac{i-\pi l y(1+n-n \sigma)}{\sigma^{2}(1-\pi) M} .
$$

Observe that an equilibrium price must involve $p<\eta \bar{\theta}$, otherwise there would be no demand for assets. Thus, equating demand and supply, we obtain

$$
\frac{\pi}{\eta \bar{\theta}} p^{2}+\frac{1-3 \pi}{2} p+\frac{1}{2}(1-\pi) \frac{i-\pi l y(1+n-n \sigma)}{\sigma^{2}(1-\pi) M}=0
$$

from which

$$
p=\frac{\eta \bar{\theta}}{2 \pi}\left\{\frac{3 \pi-1}{2} \pm\left[\frac{(3 \pi-1)^{2}}{4}-\frac{2 \pi}{\eta \bar{\theta}} \frac{i-\pi l y(1+n-n \sigma)}{\sigma^{2} M}\right]^{\frac{1}{2}}\right\} .
$$

We impose the following conditions

$$
p_{-}<\frac{i-\pi l y(1+n-n \sigma)}{\sigma^{2}(1-\pi) M} \Leftrightarrow \frac{i}{\pi l y}<1+n-n \sigma+\eta \bar{\theta} \frac{(2 \pi-1)(1-\pi) \sigma^{2} M}{\pi^{2} l y}
$$

and

$$
p_{+}>\frac{i-\pi l y(1+n-n \sigma)}{\sigma^{2}(1-\pi) M} \Leftrightarrow \frac{i}{\pi l y}<1+n-n \sigma+\eta \bar{\theta} \frac{(2 \pi-1)(1-\pi) \sigma^{2} M}{\pi^{2} l y} .
$$

For these conditions to hold, we need $\pi>\frac{1}{2}$ and $\bar{\theta}$ large enough. In particular, (15) implies that it is sufficient to have

$$
1+n-n \sigma+\eta \bar{\theta} \frac{(2 \pi-1)(1-\pi) \sigma^{2} M}{\pi^{2} l y}>1+n-n \sigma \frac{M}{M+\sigma m},
$$

that is,

$$
\bar{\theta}>\frac{1}{\eta} \frac{n m}{M+\sigma m} \frac{\pi^{2} l y}{(2 \pi-1)(1-\pi) M} .
$$

Note also that $p_{+}<\eta \bar{\theta}$ always holds. Thus, there exists a unique non-trivial equilibrium price, namely

$$
p^{*}=\frac{\eta \bar{\theta}}{2 \pi}\left\{\frac{3 \pi-1}{2}+\left[\frac{(3 \pi-1)^{2}}{4}-\frac{2 \pi}{\eta \bar{\theta}} \frac{i-\pi l y(1+n-n \sigma)}{\sigma^{2} M}\right]^{\frac{1}{2}}\right\} .
$$


Throughout, we assume that $\bar{a}\left(p^{*}, m, A\right)<1$, i.e.,

$$
p^{*}>\frac{\pi l y(1+n)-i}{\sigma(1-\pi) m} .
$$

This condition can be rewritten as

$$
\frac{\eta \bar{\theta}}{2 \pi}\left\{\frac{3 \pi-1}{2}+\left[\frac{(3 \pi-1)^{2}}{4}-\frac{2 \pi}{\eta \bar{\theta}} \frac{i-\pi l y(1+n-n \sigma)}{\sigma^{2} M}\right]^{\frac{1}{2}}\right\}>\frac{\pi l y(1+n)-i}{\sigma(1-\pi) m}
$$

or

$$
\bar{\theta}\left[\frac{(3 \pi-1)[\pi l y(1+n)-i]}{(1-\pi) m}-\frac{i-\pi l y(1+n-n \sigma)}{\sigma M}\right]>\frac{1}{\eta} \frac{2 \pi[\pi l y(1+n)-i]^{2}}{\sigma[(1-\pi) m]^{2}} .
$$

Condition (15) implies that the term in brackets that multiplies $\bar{\theta}$ is always positive as long as $\pi>\frac{1}{2}$. Thus, $\bar{a}\left(p^{*}, m, A\right)<1$ as long as

$$
\bar{\theta}>\frac{1}{\eta} \frac{\frac{2 \pi[\pi l y(1+n)-i]^{2}}{\sigma[(1-\pi) m]^{2}}}{\frac{(3 \pi-1)[\pi l y(1+n)-i]}{(1-\pi) m}-\frac{i-\pi l y(1+n-n \sigma)}{\sigma M}} .
$$

This completes our proof.

\subsection{PROOF OF PROPOSITION 2}

Regardless of the credit regime, the asset price is

$$
p^{*}=\frac{\eta \bar{\theta}}{2 \pi}\left\{\frac{3 \pi-1}{2}+\left[\frac{(3 \pi-1)^{2}}{4}-\frac{2 \pi}{\eta \bar{\theta}} \frac{i-\pi l y(1+n-n \sigma)}{\sigma^{2} M}\right]^{\frac{1}{2}}\right\} .
$$

Thus,

$$
\frac{\partial p^{*}}{\partial \eta}=\frac{1}{\eta}\left\{p^{*}+\frac{1}{2} \frac{i-\pi l y(1+n-n \sigma)}{\sigma^{2} M}\left[\frac{(3 \pi-1)^{2}}{4}-\frac{2 \pi}{\eta \bar{\theta}} \frac{i-\pi l y(1+n-n \sigma)}{\sigma^{2} M}\right]^{-\frac{1}{2}}\right\}>0 .
$$

The change in investment is computed by multiplying $i$ by the change in the measure of active firms $C$. Regardless of the credit regime, the measure of firms that obtain credit is

$$
C=1-\frac{i-\pi l y(1+n-n \sigma)}{\sigma^{2}(1-\pi) M p^{*}} .
$$

We obtain

$$
\frac{\partial I}{\partial \eta}=i \frac{\partial C}{\partial \eta}=i \frac{i-\pi l y(1+n-n \sigma)}{\sigma^{2}(1-\pi) M p^{* 2}} \frac{\partial p^{*}}{\partial \eta}>0 .
$$

In the relationship finance regime, the measure of firms that participate in credit relationships $\left(R_{r}\right)$ is equal to the measure of active firms, that is, $R_{r}=C$. Thus

$$
\frac{\partial R_{r}}{\partial \eta}=\frac{\partial C}{\partial \eta}=\frac{i-\pi l y(1+n-n \sigma)}{\sigma^{2}(1-\pi) M p^{* 2}} \frac{\partial p^{*}}{\partial \eta}>0 .
$$

In the mixed finance regime, this measure is given by

$$
R_{m}=C-\left[\widetilde{a}\left(p^{*}\right)-\bar{a}\left(p^{*}, M\right)\right] .
$$

We have

$$
R_{m}=\left\{\begin{array}{c}
C-\frac{\pi l y(1+n)-i}{\sigma(1-\pi) m} \frac{M-m}{M m} \frac{1}{p^{*}} \text { if } \bar{a}\left(p^{*}, m\right) \leq \widehat{a}\left(p^{*}\right) \\
C-\frac{1}{1-\pi}\left[\frac{(1-\sigma) \pi n y}{M-\sigma^{2} m}-\frac{\pi l y(1+n)-i}{26^{\sigma M}}\right] \frac{1}{p^{*}} \text { if } \bar{a}\left(p^{*}, m\right)>\widehat{a}\left(p^{*}\right)
\end{array} .\right.
$$


Thus,

$$
\frac{\partial R_{m}}{\partial \eta}=\left\{\begin{array}{c}
\frac{\partial C}{\partial \eta}+\frac{\pi l y(1+n)-i}{\sigma(1-\pi) m} \frac{M-m}{M m} \frac{1}{p^{* 2}} \frac{\partial p^{*}}{\partial \eta} \text { if } \bar{a}\left(p^{*}, m\right) \leq \widehat{a}\left(p^{*}\right) \\
\frac{\partial C}{\partial \eta}+\frac{1}{1-\pi}\left[\frac{(1-\sigma) \pi n y}{M-\sigma^{2} m}-\frac{\pi l y(1+n)-i}{\sigma M}\right] \frac{1}{p^{* 2}} \frac{\partial p^{*}}{\partial \eta} \text { if } \bar{a}\left(p^{*}, m\right)>\widehat{a}\left(p^{*}\right)
\end{array} .\right.
$$

Since $\frac{(1-\sigma) \pi n y}{M-\sigma^{2} m}>\frac{\pi l y(1+n)-i}{\sigma M}$ in the mixed finance regime, it is always the case that $\frac{\partial R_{m}}{\partial \eta}>0$.

In the relationship finance regime, the measure of firms that restructure is

$$
R F_{r}=\frac{\pi l y(1+n)-i}{\sigma(1-\pi) M p^{*}}-\frac{i-\pi l y(1+n-n \sigma)}{\sigma^{2}(1-\pi) M p^{*}}=\frac{\pi l y(1+n+\sigma)-i(1+\sigma)}{\sigma^{2}(1-\pi) M p^{*}} .
$$

We obtain

$$
\frac{\partial R F_{r}}{\partial \eta}=-\frac{\pi l y(1+n+\sigma)-i(1+\sigma)}{\sigma^{2}(1-\pi) M p^{* 2}} \frac{\partial p^{*}}{\partial \eta} .
$$

Condition (15) implies that this derivative is negative. In turn, in the mixed finance regime, the measure of firms that restructure is

$$
R F_{m}=\widetilde{a}\left(p^{*}\right)-\frac{i-\pi l y(1+n-n \sigma)}{\sigma^{2}(1-\pi) M p^{*}} .
$$

We have

$$
R F_{m}=\left\{\begin{array}{c}
{\left[\frac{\pi l y(1+n)-i}{\sigma(1-\pi) m}-\frac{i-\pi l y(1+n-n \sigma)}{\sigma^{2}(1-\pi) M}\right] \frac{1}{p^{*}} \text { if } \bar{a}\left(p^{*}, m\right) \leq \widehat{a}\left(p^{*}\right)} \\
{\left[\frac{(1-\sigma) \pi n y}{(1-\pi)\left(M-\sigma^{2} m\right)}-\frac{i-\pi l y(1+n-n \sigma)}{\sigma^{2}(1-\pi) M}\right] \frac{1}{p^{*}} \text { if } \bar{a}\left(p^{*}, m\right)>\widehat{a}\left(p^{*}\right)}
\end{array} .\right.
$$

and, hence,

$$
\frac{\partial R F_{m}}{\partial \eta}=\left\{\begin{array}{c}
-\left[\frac{\pi l y(1+n)-i}{\sigma(1-\pi) m}-\frac{i-\pi l y(1+n-n \sigma)}{\sigma^{2}(1-\pi) M}\right] \frac{1}{p^{* 2}} \frac{\partial p^{*}}{\partial \eta} \text { if } \bar{a}\left(p^{*}, m\right) \leq \widehat{a}\left(p^{*}\right) \\
-\left[\frac{(1-\sigma) \pi n y}{(1-\pi)\left(M-\sigma^{2} m\right)}-\frac{i-\pi l y(1+n-n \sigma)}{\sigma^{2}(1-\pi) M}\right] \frac{1}{p^{* 2}} \frac{\partial p^{*}}{\partial \eta} \text { if if } \bar{a}\left(p^{*}, m\right)>\widehat{a}\left(p^{*}\right)
\end{array} .\right.
$$

Condition (15) implies that $\frac{\pi l y(1+n)-i}{\sigma(1-\pi) m}-\frac{i-\pi l y(1+n-n \sigma)}{\sigma^{2}(1-\pi) M}>0$ and $\frac{(1-\sigma) \pi n y}{(1-\pi)\left(M-\sigma^{2} m\right)}-\frac{i-\pi l y(1+n-n \sigma)}{\sigma^{2}(1-\pi) M}>0$. Thus, it is always the case that $\frac{\partial R F_{m}}{\partial \eta}<0$.

Finally, output is given by

$$
Y_{i}=\omega+\left(\pi y+\frac{1-\pi}{2} \frac{(\bar{\theta} \eta)^{2}-\left(p^{*}\right)^{2}}{\bar{\theta} \eta}-i\right) C+R F_{i}(1-\sigma) \pi n y,
$$

where $i \in\{r, m\}$ identifies the relationship or mixed credit regime. We obtain,

$$
\frac{\partial Y_{i}}{\partial \eta}=\frac{\partial C}{\partial \eta}(\pi y-i)+\frac{\partial R F_{i}}{\partial \eta}(1-\sigma) \pi n y+\frac{1-\pi}{2} \frac{1}{\bar{\theta} \eta}\left\{\frac{\partial C}{\partial \eta}\left[(\bar{\theta} \eta)^{2}-\left(p^{*}\right)^{2}\right]+C\left[\frac{\left(p^{*}\right)^{2}+(\bar{\theta} \eta)^{2}}{\eta}-2 p^{*} \frac{\partial p^{*}}{\partial \eta}\right]\right\} .
$$

In general, the effect of the shock on output is ambiguous since $\frac{\partial C}{\partial \eta}>0$ and $\frac{\partial R F_{i}}{\partial \eta}<0$.

\subsection{PROOF OF PROPOSITION 3}

If $\bar{a}\left(p^{*}, m\right) \leq \widehat{a}\left(p^{*}\right)$, we have

$$
\begin{aligned}
\frac{\partial\left(\frac{R_{c}^{\bar{a}} \leq \widehat{a}}{C}\right)}{\partial \eta} & =\frac{C \frac{\partial R_{c}^{\bar{a} \leq \widehat{a}}}{\partial \eta}-R_{c}^{\bar{a} \leq \widehat{a}} \frac{\partial C}{\partial \eta}}{C^{2}}= \\
& =\frac{C\left(\frac{\partial C}{\partial \eta}+\left[\frac{\pi l y(1+n)-i}{\sigma(1-\pi) m}-\frac{\pi l y(1+n)-i}{\sigma(1-\pi) M}\right] \frac{1}{p^{* 2}} \frac{\partial p^{*}}{\partial \eta}\right)-\left(C-\left[\frac{\pi l y(1+n)-i}{\sigma(1-\pi) m}-\frac{\pi l y(1+n)-i}{\sigma(1-\pi) M}\right] \frac{1}{p^{*}}\right) \frac{\partial C}{\partial \eta}}{C^{2}}(70)
\end{aligned}
$$


This can be rewritten as

$$
\frac{\partial\left(\frac{R_{c}^{\pi \leq \hat{a}}}{C}\right)}{\partial \eta}=\frac{C\left[\frac{\pi l y(1+n)-i}{\sigma(1-\pi) m}-\frac{\pi l y(1+n)-i}{\sigma(1-\pi) M}\right] \frac{1}{p^{* 2}} \frac{\partial p^{*}}{\partial \eta}+\left[\frac{\pi l y(1+n)-i}{\sigma(1-\pi) m}-\frac{\pi l y(1+n)-i}{\sigma(1-\pi) M}\right] \frac{1}{p^{*}} \frac{\partial C}{\partial \eta}}{C^{2}}
$$

or also

$$
\begin{aligned}
& \frac{\partial\left(\frac{R_{c}^{\bar{a} \leq \widehat{a}}}{C}\right)}{\partial \eta}=\frac{\left[\frac{\pi l y(1+n)-i}{\sigma(1-\pi) m}-\frac{\pi l y(1+n)-i}{\sigma(1-\pi) M}\right] \frac{1}{\left(p^{*}\right)^{2}} \frac{\partial p^{*}}{\partial \eta}}{C^{2}}, \\
& \frac{\partial\left(\frac{R_{c}^{\bar{a}} \leq \widehat{a}}{C}\right)}{\partial \eta}=\frac{C-R_{c}^{\bar{a} \leq \widehat{a}}}{C^{2}} \frac{1}{p^{*}} \frac{\partial p^{*}}{\partial \eta}>0 .
\end{aligned}
$$

If $\bar{a}\left(p^{*}, m\right)>\widehat{a}\left(p^{*}\right)$, we have

$$
\begin{aligned}
\frac{\partial\left(\frac{R_{c}^{\bar{a}>\widehat{a}}}{C}\right)}{\partial \eta} & =\frac{C \frac{\partial R_{c}^{\bar{a}>\widehat{a}}}{\partial \eta}-R_{c}^{\bar{a}>\widehat{a}} \frac{\partial C}{\partial \eta}}{C^{2}}= \\
& =\frac{C\left(\frac{\partial C}{\partial \eta}+\left[\frac{(1-\sigma) \pi n y}{(1-\pi)\left(M-\sigma^{2} m\right)}-\frac{\pi l y(1+n)-i}{\sigma(1-\pi) M}\right] \frac{1}{p^{* 2}} \frac{\partial p^{*}}{\partial \eta}\right)-\left(C-\left[\frac{(1-\sigma) \pi n y}{(1-\pi)\left(M-\sigma^{2} m\right)}-\frac{\pi l y(1+n)-i}{\sigma(1-\pi) M}\right] \frac{1}{p^{*}}\right) \frac{\partial C}{\partial \eta}}{C^{2}}
\end{aligned}
$$

This can be rewritten as

$$
\frac{\partial\left(\frac{R_{c}^{\bar{a}>\widehat{a}}}{C}\right)}{\partial \eta}=\frac{C\left[\frac{(1-\sigma) \pi n y}{(1-\pi)\left(M-\sigma^{2} m\right)}-\frac{\pi l y(1+n)-i}{\sigma(1-\pi) M}\right] \frac{1}{p^{* 2}} \frac{\partial p^{*}}{\partial \eta}+\left[\frac{(1-\sigma) \pi n y}{(1-\pi)\left(M-\sigma^{2} m\right)}-\frac{\pi l y(1+n)-i}{\sigma(1-\pi) M}\right] \frac{1}{p^{*}} \frac{\partial C}{\partial \eta}}{C^{2}}
$$

or also

$$
\begin{aligned}
& \frac{\partial\left(\frac{R_{c}^{\bar{a}>\widehat{a}}}{C}\right)}{\partial \eta}=\frac{\left[\frac{(1-\sigma) \pi n y}{(1-\pi)\left(M-\sigma^{2} m\right)}-\frac{\pi l y(1+n)-i}{\sigma(1-\pi) M}\right] \frac{1}{p^{*}} \frac{1}{p^{*}} \frac{\partial p^{*}}{\partial \eta}}{C^{2}} \\
& \frac{\partial\left(\frac{R_{c}^{\bar{a}>\widehat{a}}}{C}\right)}{\partial \eta}=\frac{C-R_{c}^{\bar{a}>\widehat{a}}}{C^{2}} \frac{1}{p^{*}} \frac{\partial p^{*}}{\partial \eta}>0 .
\end{aligned}
$$

\subsection{PROOF OF PROPOSITION 4}

PROPOSITION 4: In both the relationship finance regime and the mixed finance regime, a negative productivity shock reduces the asset price, total investment, the measure of firms that have access to credit, and the measure of firms that engage in credit relationships. A negative productivity shock also reduces the measure of restructuring firms and output. However, the drop in the measure of restructuring firms is smaller than the drop in total investment.

Regardless of the credit regime, the asset price is

$$
\begin{gathered}
p=\frac{\eta \bar{\theta}}{2 \pi}\left\{\frac{3 \pi-1}{2}+\left[\left(\frac{3 \pi-1}{2}\right)^{2}-\frac{2 \pi}{\eta \bar{\theta}} \frac{i-\pi l y(1+n-n \sigma)}{\sigma^{2} M}\right]^{\frac{1}{2}}\right\} . \\
\frac{\partial p}{\partial y}=\frac{1}{2 y}\left[\left(\frac{3 \pi-1}{2}\right)^{2}-\frac{2 \pi}{\eta \bar{\theta}} \frac{i-\pi l y(1+n-n \sigma)}{\sigma^{2} M}\right]^{-\frac{1}{2}} \frac{\pi l y(1+n-n \sigma)}{\sigma^{2} M}>0 .
\end{gathered}
$$

The change in investment is computed by multiplying $i$ by the change in the measure of active firms $C$. Regardless of the credit regime, the measure of firms that obtain credit is

$$
C=1-\frac{i-\pi l y(1+n-n \sigma)}{\sigma_{2}^{2}(1-\pi) M p}
$$


We obtain

$$
\frac{\partial I}{\partial y}=i \frac{\partial C}{\partial y}=i \frac{\pi l y(1+n-n \sigma)+[i-\pi l y(1+n-n \sigma)] \frac{\partial p}{\partial y} \frac{y}{p}}{\sigma^{2}(1-\pi) M p y}>0 .
$$

In the relationship finance regime, the measure of firms that participate in credit relationships $\left(R_{r}\right)$ is equal to the measure of active firms, that is, $R_{r}=C$. Thus

$$
\frac{\partial R_{r}}{\partial y}=\frac{\partial C}{\partial y}=\frac{\pi l y(1+n-n \sigma)+[i-\pi l y(1+n-n \sigma)] \frac{\partial p}{\partial y} \frac{y}{p}}{\sigma^{2}(1-\pi) M p y}>0 .
$$

In the mixed finance regime, this measure is given by (remember that $\widehat{a}(p)<\bar{a}(p, m)$ in the mixed finance regime)

$$
R_{m}=C-[\widehat{a}(p)-\bar{a}(p, M)]=C-\frac{1}{1-\pi}\left[\frac{(1-\sigma) \pi n y}{M-\sigma^{2} m}-\frac{\pi l y(1+n)-i}{\sigma M}\right] \frac{1}{p} .
$$

Thus,

$$
\frac{\partial R_{m}}{\partial y}=\frac{\partial C}{\partial y}-\frac{1}{1-\pi} \frac{\partial\left[\frac{(1-\sigma) \pi n y}{M-\sigma^{2} m}-\frac{\pi l y(1+n)-i}{\sigma M}\right] \frac{1}{p}}{\partial y} .
$$

After some algebraic manipulation, we obtain

$$
\frac{\partial R_{m}}{\partial y}=\frac{a}{(p, M, A)+\widehat{a}(p)-\bar{a}(p, M)} \frac{\partial p}{\partial y}+\frac{\pi}{1-\pi} \frac{1+n+n \sigma}{\sigma^{2} M p}[\underbrace{l-\frac{(1-\sigma) n \sigma^{2} M}{\left(M-\sigma^{2} m\right)(1+n+n \sigma)}}_{\text {positive since } l>\frac{1}{1+\sigma}}]>0 .
$$

It remains to consider the effect of the shock on the measure of firms that restructure and on the output. We start with restructuring. In the relationship finance regime, the measure of firms that restructure is

$$
R F_{r}=\bar{a}(p, M)-\underline{a}(p, M, A)=\frac{\pi l y(1+n+\sigma)-i(1+\sigma)}{\sigma^{2}(1-\pi) M p} .
$$

We obtain

$$
\frac{\partial R F_{r}}{\partial y}=\frac{\partial \bar{a}(p, M)}{\partial y}-\frac{\partial \underline{a}(p, M, A)}{\partial y} .
$$

We can rewrite the above derivative as

$$
\begin{aligned}
& \frac{\partial R F_{r}}{\partial y}=-\frac{\bar{a}(p, M)}{y}\left[\frac{y}{p} \frac{\partial p}{\partial y}-\frac{\pi l y(1+n)}{\pi l y(1+n)-i}\right]+\frac{a(p, M, A)}{y}\left[\frac{y}{p} \frac{\partial p}{\partial y}+\frac{\pi l y(1+n-n \sigma)}{i-\pi l y(1+n-n \sigma)}\right] . \\
& \frac{\frac{\partial R F_{r}}{\partial y}}{R F_{r}}<\frac{\frac{\partial I}{\partial y}}{I} \\
& \frac{-\frac{\bar{a}(p, M)}{y}\left[\frac{y}{p} \frac{\partial p}{\partial y}-\frac{\pi l y(1+n)}{\pi l y(1+n)-i}\right]+\frac{\underline{a}(p, M, A)}{y}\left[\frac{y}{p} \frac{\partial p}{\partial y}+\frac{\pi l y(1+n-n \sigma)}{i-\pi l y(1+n-n \sigma)}\right]}{\bar{a}(p, M)-\underline{a}(p, M, A)}<\frac{\frac{\underline{\underline{a}(p, M, A)}}{y}\left[\frac{y}{p} \frac{\partial p}{\partial y}+\frac{\pi l y(1+n-n \sigma)}{i-\pi l y(1+n-n \sigma)}\right]}{1-\underline{a}(p, M, A)}
\end{aligned}
$$

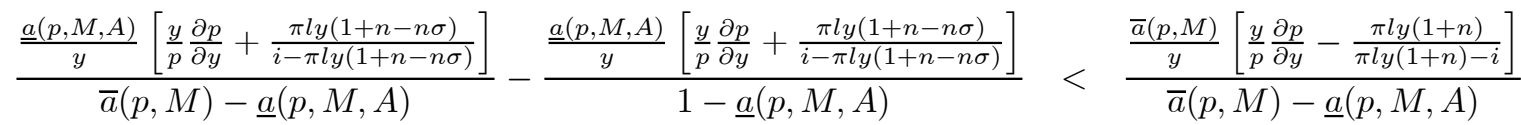

$$
\begin{aligned}
& \frac{\underline{a}(p, M, A)}{\bar{a}(p, M)} \frac{1-\bar{a}(p, M)}{1-\underline{a}(p, M, A)}\left[\frac{y}{p} \frac{\partial p}{\partial y}+\frac{\pi l y(1+n-n \sigma)}{i-\pi l y(1+n-n \sigma)}\right]<\left[\frac{y}{p} \frac{\partial p}{\partial y}-\frac{\pi l y(1+n)}{\pi l y(1+n)-i}\right] \\
& \frac{y}{p} \frac{\partial p}{\partial y}>\left[\begin{array}{c}
\frac{a(p, M, A)[1-\bar{a}(p, M)]}{\bar{a}(p, M)-a(p, M, A)} \frac{\pi l y(1+n-n \sigma)}{i-\pi l y(1+n-n \sigma)}+ \\
\frac{\bar{a}(p, M)[1-\underline{a}(p, M, A)]}{\bar{a}(p, M)-\underline{a}(p, M, A)} \frac{\pi l y(1+n)}{\pi l y(1+n)-i}
\end{array}\right]
\end{aligned}
$$




$$
\frac{i}{\pi l y} \in\left[1+n-\frac{n \sigma}{l(1+\sigma)}, 1+n-\frac{n \sigma M(1-\sigma)}{l\left(M-\sigma^{2} m\right)}\right)
$$

Consider now the mixed finance regime. The measure of firms that restructure is

$$
R F_{m}=\widehat{a}(p)-\underline{a}(p, M, A)=\frac{(1-\sigma) \pi n y}{(1-\pi)\left(M-\sigma^{2} m\right) p}-\frac{i-\pi l y(1+n-n \sigma)}{\sigma^{2}(1-\pi) M p} .
$$

We obtain

$$
\frac{\partial R F_{m}}{\partial y}=\frac{\partial \widehat{a}(p)}{\partial y}-\frac{\partial \underline{a}(p, M, A)}{\partial y} .
$$

We can rewrite the above derivative as

$$
\frac{\partial R F_{m}}{\partial y}=-\frac{\widehat{a}(p)}{y}\left[\frac{y}{p} \frac{\partial p}{\partial y}-1\right]+\frac{a(p, M, A)}{y}\left[\frac{y}{p} \frac{\partial p}{\partial y}+\frac{\pi l y(1+n-n \sigma)}{i-\pi l y(1+n-n \sigma)}\right] .
$$

As in the relationship finance regime, note that the second term on the right hand side is always positive, while the first term on the right hand is negative if and only if

$$
\frac{y}{p} \frac{\partial p}{\partial y}>1
$$

After some algebraic manipulation, we can rewrite this inequality as

$$
\eta \bar{\theta}<\frac{8 \pi}{(3 \pi-1)^{2}}\left\{\frac{i-\pi l y(1+n-n \sigma)}{\sigma^{2} M}+\frac{\left[\frac{1}{2} \frac{\pi l y(1+n-n \sigma)}{\sigma^{2} M}\right]^{2}}{\frac{i}{\sigma^{2} M}}\right\} .
$$

The requirement that $\bar{a}(p, m)<1$ implies that there exists a positive region of parameters where the above condition hold if and only if

$$
\frac{8 \pi(1-\pi)}{(3 \pi-1)^{2}}\left\{\underline{a}(p, M, A)+\frac{1}{4} \frac{1}{i} \frac{[\pi l y(1+n-n \sigma)]^{2}}{\sigma^{2}(1-\pi) M p}\right\}>\frac{[\bar{a}(p, m)]^{2}}{(3 \pi-1) \bar{a}(p, m)-(1-\pi) \underline{a}(p, M, A)} .
$$

The mixed finance regime occurs for all $\frac{i}{\pi l y} \in\left[1+n-\frac{n \sigma M(1-\sigma)}{l\left(M-\sigma^{2} m\right)}, 1+n-\frac{n \sigma M}{M+\sigma m}\right]$ if $\frac{M-m}{m}<\frac{1-\sigma^{2}}{\sigma}$ and $l \in\left[\frac{1}{1+\sigma}, \frac{(M+\sigma m)(1-\sigma)}{M-\sigma^{2} m}\right]$. Let then $l \approx \frac{1}{1+\sigma}$ and $\frac{i}{\pi l y} \approx 1+n-\frac{n \sigma M\left(1-\sigma^{2}\right)}{\left(M-\sigma^{2} m\right)}$. This implies $\underline{a}(p, M, A) \approx$ $\frac{\pi l y n \sigma(M-m)}{(1-\pi) M\left(M-\sigma^{2} m\right) p}$ and $\bar{a}(p, m)=\frac{\pi l y n \sigma M\left(1-\sigma^{2}\right)}{\sigma(1-\pi) M\left(M-\sigma^{2} m\right) p}$. If we further let $M \approx m$, we can rewrite (78) as

$$
\frac{1+n-n \sigma}{n \sigma^{2}}>\frac{3 \pi-1}{2 \pi(1-\pi)} .
$$

This condition can be satisfied if $\pi, \sigma$ and $n$ are not too large. Lastly, we look at the effect of the productivity shock on output. Output is given by

$$
Y_{i}=\omega+[\pi y-i+(1-\pi) \operatorname{Pr}(\theta \eta>p) E(\theta \eta \mid \theta \eta>p)] C+R F_{i}(1-\sigma) \pi n y,
$$

where $i \in\{r, m\}$ identifies the relationship or mixed credit regime. We obtain.

$$
\frac{\partial Y_{i}}{\partial y}=\frac{\partial C}{\partial y}(\pi y-i)+\frac{\partial R F_{i}}{\partial y}(1-\sigma) \pi n y+\pi\left[C+R F_{i}(1-\sigma) n\right]+(1-\pi) \frac{\partial \operatorname{Pr}(\theta \eta>p) E(\theta \eta \mid \theta \eta>p) C}{\partial y} .
$$




\subsection{PROOF OF PROPOSITION 5}

The demand for assets is the same as in Proposition 1, that is,

$$
D(p)=\max \left\{[1-\underline{a}(p, M, A)] \pi\left(1-\frac{p}{\eta \bar{\theta}}\right), 0\right\} .
$$

The supply of assets now depends on the degree of restructuring, and is given by

$$
S(p)=\max \left\{(1-\pi)\left[\int_{\underline{a}(p, M, A)}^{\max \{\bar{a}(p, M), \widetilde{a}(p)\}}[(1-\sigma) \phi+\sigma] a d a+\int_{\max \{\bar{a}(p, M), \widetilde{a}(p)\}}^{1} a d a\right], 0\right\} .
$$

The supply of assets can be rewritten as

$$
S(p)=\max \left\{\frac{1-\pi}{2}\left\{[(1-\sigma) \phi+\sigma]\left[a_{+}^{2}-(\underline{a}(p, M, A))^{2}\right]+1-a_{+}^{2}\right\}, 0\right\},
$$

where $a_{+} \equiv \max \{\bar{a}(p, M), \widetilde{a}(p)\}$. As before, we restrict attention to non-trivial equilibria in which $\underline{a}(p, M, A)<$ 1 and $p<\eta \bar{\theta}$. This implies

$$
S(p)=\frac{1-\pi}{2} \sigma\left[1-(\underline{a}(p, M, A))^{2}\right]+\frac{1-\pi}{2}(1-\sigma)\left\{\phi\left[a_{+}^{2}-(\underline{a}(p, M, A))^{2}\right]+1-a_{+}^{2}\right\} .
$$

Equating demand and supply, we obtain

$[1-\underline{a}(p, M, A)] \pi\left(1-\frac{p}{\eta \bar{\theta}}\right)=\frac{1-\pi}{2} \sigma\left[1-(\underline{a}(p, M, A))^{2}\right]+\frac{1-\pi}{2}(1-\sigma)\left\{\phi\left[a_{+}^{2}-(\underline{a}(p, M, A))^{2}\right]+1-a_{+}^{2}\right\}$.

First, note that, at any given price, $\sigma=1$ implies a supply of assets equal to that in Proposition 1 , while $\sigma=0$ implies a supply of assets that is below the supply in Proposition 1 (since $\phi<1$ ). Thus, the equilibrium price under asset heterogeneity must be larger than the equilibrium price when assets have the same degree of redeployability. Note also that the fact that $\phi<1$ only impacts the distribution of firms through a different price. In fact, $(\underline{a}(p, M, A))^{2}$ and $a_{+} \equiv \max \{\bar{a}(p, M), \widetilde{a}(p)\}$ do not depend directly on $\phi$. This implies that, in order to compare the distribution under asset heterogeneity with the distribution in Proposition 1, we only need to examine how the asset price affects the distribution of firms. We observe that, since the asset price is higher under asset heterogeneity, there will be more active firms, more investment, more credit relationships and less restructuring.

It remains to be examined the impact of a negative collateral shock, i.e., a decrease in $\eta$. First, a decrease in $\eta$ leads to a reduction of the demand for assets at any price level, but it does not affect the position of the supply curve. In other words, the movement on the supply of assets is a movement along the supply curve, driven by the reduction of the asset price. If assets are homogeneous, the only impact of a reduction of the asset price on the supply of assets is caused by a reduction in the number of firms that have access to credit. If, instead, assets are heterogeneous, the reduction of the asset price not only reduces the number of firms that have access to credit (and by the same amount that it does so under homogeneous assets, since the expression for $\underline{a}(p, M, A)$ only depends on $\eta$ through the asset price), but it also reduces the supply of assets due to the increase in restructuring (this is so because $a_{+} \equiv \max \{\bar{a}(p, M), \widetilde{a}(p)\}$ is decreasing in $p$ ). As a result, it must be the case that a negative collateral shock has a smaller impact on the asset price when assets are heterogeneous. Finally, since the change in $\eta$ only affects the distribution of firms through the change in asset price, it must be the that all the effects in Proposition 3 are dampened when assets are heterogeneous. 


\subsection{PROOFS OF SECTION 5}

Policy 1 The demand for assets is

$$
D(p)=\max \left\{[1-\underline{a}(p, M, A)] \pi\left(1-\frac{p-\tau}{\eta \bar{\theta}}\right), 0\right\},
$$

while the supply of assets is

$$
S(p)=\max \left\{\frac{1}{2}(1-\pi)\left[1-(\underline{a}(p, M, A))^{2}\right], 0\right\} .
$$

Equating demand and supply, we obtain

$$
\frac{\pi}{\eta \bar{\theta}} p^{2}+\left(\frac{1-3 \pi}{2}-\frac{\pi}{\eta \bar{\theta}} \tau\right) p+\frac{1}{2}(1-\pi) \frac{i-\pi l y(1+n-n \sigma)}{\sigma^{2}(1-\pi) M}=0 .
$$

from which

$$
p=\frac{-\left(\frac{1-3 \pi}{2}-\frac{\pi}{\eta \bar{\theta}} \tau\right) \pm\left[\left(\frac{1-3 \pi}{2}-\frac{\pi}{\eta \bar{\theta}} \tau\right)^{2}-\frac{1}{\eta \bar{\theta}} 2 \pi(1-\pi) \frac{i-\pi l y(1+n-n \sigma)}{\sigma^{2}(1-\pi) M}\right]^{\frac{1}{2}}}{2 \frac{\pi}{\eta \bar{\theta}}} .
$$

We impose the following conditions

$$
p_{-}<\frac{i-\pi l y(1+n-n \sigma)}{\sigma^{2}(1-\pi) M} \Leftrightarrow \frac{i}{\pi l y}<1+n-n \sigma+\eta \bar{\theta} \frac{\left(2 \pi-1+\frac{\pi}{\eta \bar{\theta}} \tau\right)(1-\pi) \sigma^{2} M}{\pi^{2} l y}
$$

and

$$
p_{+}>\frac{i-\pi l y(1+n-n \sigma)}{\sigma^{2}(1-\pi) M} \Leftrightarrow \frac{i}{\pi l y}<1+n-n \sigma+\eta \bar{\theta} \frac{\left(2 \pi-1+\frac{\pi}{\eta \bar{\theta}} \tau\right)(1-\pi) \sigma^{2} M}{\pi^{2} l y} .
$$

For these conditions to hold, we need $2 \pi-1+\frac{\pi}{\eta \bar{\theta}} \tau>0$ and $\bar{\theta}$ large enough. Note also that $p_{+}<\eta \bar{\theta}$ always holds. Thus, there exists a unique non-trivial equilibrium price, namely

$$
p^{*}=\frac{1}{\frac{2 \pi}{\eta \bar{\theta}}}\left\{\pi-\frac{1-\pi}{2}+\frac{\pi}{\eta \bar{\theta}} \tau+\left[\left(\pi-\frac{1-\pi}{2}+\frac{\pi}{\eta \bar{\theta}} \tau\right)^{2}-\frac{2 \pi}{\eta \bar{\theta}} \frac{i-\pi l y(1+n-n \sigma)}{\sigma^{2} M}\right]^{\frac{1}{2}}\right\} .
$$

Again, we assume that $\bar{a}\left(p^{*}, m\right)<1$, which is always satisfied as long as $\pi>\frac{1}{2}$ and $\bar{\theta}$ is large enough. Clearly, an increase in $\tau$ leads to an increase in the asset price.

Under a transfer $\tau$, output is given by

$$
Y(\tau)=\omega+C(\tau)\left[\pi y+\frac{1-\pi}{2} \frac{(\bar{\theta} \eta)^{2}-\left(p^{*}(\tau)-\tau\right)^{2}}{\bar{\theta} \eta}-i\right]+R F(\tau)(1-\sigma) \pi n y,
$$

where

$$
C(\tau)=1-\underline{a}\left(p^{*}(\tau), M, A\right)
$$

is the measure of firms with access to credit and

$$
R F(\tau)=\bar{a}\left(p^{*}(\tau), M\right)-\underline{a}\left(p^{*}(\tau), M, A\right)
$$

is the measure of firms that restructure their activity. The derivatives (22)-(24) in the text can be obtained using (90)-(93). 
Policy 2 The demand for assets is

$$
D(p)=\max \left\{\left(1-\underline{a}_{G}\right) \pi\left(1-\frac{p}{\eta \bar{\theta}}\right), 0\right\},
$$

while the supply of assets is

$$
S(p)=\max \left\{\frac{1}{2}(1-\pi)\left(1-\underline{a}_{G}^{2}\right), 0\right\} .
$$

Equating demand and supply, we obtain

$$
\left(1-\underline{a}_{G}\right) \pi\left(1-\frac{p}{\eta \bar{\theta}}\right)=\frac{1}{2}(1-\pi)\left(1-\underline{a}_{G}^{2}\right),
$$

which can be rewritten as

$$
p^{*}\left(\underline{a}_{G}\right)=\frac{\eta \bar{\theta}}{2 \pi}\left[3 \pi-1-(1-\pi) \underline{a}_{G}\right] .
$$

We assume that $\bar{a}\left(p^{*}\left(\underline{a}_{G}\right), m\right)<1$, which is always satisfied as long as $\pi>\frac{1}{2}$ and $\bar{\theta}$ is large enough. Clearly, a reduction in $\underline{a}_{G}$ leads to an increase in the asset price.

Output is

$$
Y\left(\underline{a}_{G}\right)=\omega+C\left(\underline{a}_{G}\right)\left[\pi y+\frac{1-\pi}{2} \frac{(\bar{\theta} \eta)^{2}-\left(p^{*}\left(\underline{a}_{G}\right)\right)^{2}}{\bar{\theta} \eta}-i\right]+R F\left(\underline{a}_{G}\right)(1-\sigma) \pi n y,
$$

where $C\left(\underline{a}_{G}\right)=1-\underline{a}_{G}$ is the measure of firms with access to credit and $R F\left(\underline{a}_{G}\right)=\bar{a}\left(p^{*}\left(\underline{a}_{G}\right), M\right)-\underline{a}_{G}$ is the measure of firms that restructure their activity.

\section{References}

Aghion, P. and P. Bolton (1992) "An incomplete contracts approach to financial contracting," Review of Economic Studies 59, 3, 473-494.

Barlevy, G. (2003) "Credit market frictions and the allocation of resources over the business cycle," Journal of Monetary Economics 50, 8, 1795-1818.

Berger, A. N. and G. F. Udell (2002) "Small business credit availability and relationship lending: The importance of bank organisational structure," Economic Journal 102, 2, 32-53.

Berger, A. N. and G. Udell (1998) "The economics of small business finance: The roles of private equity and debt markets in the financial growth cycle," Journal of Banking and Finance 22, 6-8, 613-673.

Berger, A. and G. Udell (1995) "Relationship lending and lines of credit in small firm finance," Journal of Business 68, 3, 351-381.

Berlin, M. and A. Butler (2002) "Collateral and competition," Working paper 02-22, Federal Reserve Bank of Philadelphia.

Bhattacharya, S. and G. Chiesa (1995) "Proprietary information, financial intermediation, and research incentives," Journal of Financial Intermediation 4, 4, 328-357.

Caballero, R. and M. Hammour (2005) "The costs of recessions revisited: a reverse-liquidationist view," Review of Economic Studies 72, 2, 313-341. 
Caballero, R. and P. Kurat (2009) "Public-private partnerships for liquidity provision," Unpublished working paper, MIT.

Carpenter, R. and B. Petersen (2002) "Capital market imperfections, high-tech investment, and new equity financing," Economic Journal 102, 2, 54-72.

Dell' Ariccia, G. and P. Garibaldi (2004) "Gross credit flows," Review of Economic Studies 72, 3, 665-685.

Dell' Ariccia, G. and P. Garibaldi (2001) "Bank lending and interest rate changes in a dynamic matching model," International Monetary Fund, Working Paper 98/93.

Den Haan, W. J., G. Ramey and J. Watson (2003) "Liquidity flows and fragility of business enterprises," Journal of Monetary Economics 50, 6, 1215-1241.

Diamond, D. and R. Rajan (2001) "Liquidity risk, liquidity creation and financial fragility: a theory of banking," Journal of Political Economy 109, 2, 287-327.

Gertler, M. and P. Karadi (2011) "A model of unconventional monetary policy," Journal of Monetary Economics $58,1,17-34$.

Gertler, M. and N. Kiyotaki (2011) "Financial intermediation and credit policy in business cycle analysis," Working paper, New York University.

Goodacre, A. and I. Tonks (1995) "Finance and technological change," in P. Stoneman, ed.: Handbook of the economics of innovation and technological change. Blackwell, Oxford.

Guiso, L. and R. Minetti (2010) "The structure of multiple credit relationships: Evidence from U.S. firms," Journal of Money, Credit and Banking, 42, 6, 1037-1071.

Habib, M. A. and D. B. Johnsen (1999) "The financing and redeployment of specific assets," Journal of Finance 54, 2, 693-720.

Holmstrom, B., and J. Tirole (1997) "Financial intermediation, loanable funds and the real sector," Quarterly Journal of Economics 112, 3, 663-691.

Kiyotaki, N., and J. Moore (1997) "Credit cycles," Journal of Political Economy 105, 2, 211-248.

Krishnamurthy, A. (2010) "Amplification mechanisms in liquidity crises," American Economic Journal: Macroeconomics, 2, 3, 1-30.

Manove, M. and A. J. Padilla (1999) "Banking (conservatively) with optimists," Rand Journal of Economics $30,2,324-350$.

Manove, M., A. J. Padilla and M. Pagano (2001) "Collateral versus project screening: a model of lazy banks," Rand Journal of Economics, 32, 4, 726-744.

Rajan, R. (1992) "Insiders and outsiders: the choice between informed and arm's length debt," Journal of Finance 47, 4, 1367-1400.

Rajan, R. and L. Zingales (2001) "Financial systems, industrial structure, and growth," Oxford Review of Economic Policy 17, 4, 467-482.

Ramey, G. (2004) "Financial intermediation in a restructuring economy," Unpublished working paper, University of California San Diego. 


\section{SUPPLEMENT - EQUILIBRIUM CHARACTERIZATION}

Throughout, to simplify notation, we drop the dependence of thresholds on the price level. A number of thresholds matter in the description of the equilibrium. First, a lender does not implement the action that allows innovation if and only if

$$
a \geq \bar{a}(m) \equiv \frac{\pi l y(1+n)-i}{\sigma(1-\pi) m p} .
$$

In turn, a lender can always be induced to implement the action that allows innovation if and only if

$$
a<\bar{a}(M) \equiv \frac{\pi l y(1+n)-i}{\sigma(1-\pi) M p} .
$$

Finally, if

$$
\bar{a}(m)>a \geq \bar{a}(M),
$$

a lender can only be induced to implement the action that allows innovation if the borrower chooses transactional funding. We also need to describe the thresholds that allow the borrower to obtain credit. Let

$$
\underline{a}(\mu, A) \equiv \frac{i-\pi l y(1+n-n \sigma)}{\sigma^{2}(1-\pi) \mu p} .
$$

Then, if the lender implements the action that allows innovation, the borrower obtains credit if and only if $a>\underline{a}(\mu, A)$, where $\mu \in\{M, m\}$ denotes the choice between transactional and relational funding. Now, let

$$
\underline{a}(\mu, \bar{A}) \equiv \frac{i-\pi l y}{(1-\pi) \mu p} .
$$

If the lender does not implement the action that allows innovation, the borrower obtains credit if and only if $a>\underline{a}(\mu, \bar{A})$. where $\mu \in\{M, m\}$ is as above.

We assume that, irrespective of $\mu \in\{M, m\}$, borrowers with small enough $a$ do not obtain credit. This implies $\underline{a}(\mu, \bar{A})>\underline{a}(\mu, A)>0$, i.e.,

$$
A 1: \frac{i}{\pi l y}>1+n-n \sigma .
$$

Throughout, we identify outcomes by the pair $(\mu, \alpha)$, where $\mu \in\{M, m\}$ denotes the choice of the borrower between relational and transactional funding, and $\alpha \in\{A, \bar{A}\}$ denotes the behavior of the lender, that is, whether she chooses to implement or not the action that allows innovation.

In what follows we characterize equilibrium behavior for all values of $\frac{i}{\pi l y}$ consistent with $A 1$. We start with

$$
\frac{i}{\pi l y} \geq 1+n
$$

In this case, $\bar{a}(m) \leq 0$ and innovation never occurs. Thus, it must be that the borrower chooses relational funding. Moreover, the borrower obtains credit if and only if $a>\underline{a}(M, \bar{A})$.

Henceforth, we assume that

$$
\frac{i}{\pi l y} \in(1+n-n \sigma, 1+n),
$$

and proceed in steps, initially restricting attention to firms with $a \geq \bar{a}(m)$. 


\subsection{1 $a \geq \bar{a}(m):$ collateral rich firms}

If $a \geq \bar{a}(m)$, the lender never implements the action that allows innovation. Thus, the borrower always chooses relational funding. Credit is available for all firms in this range if and only if $\bar{a}(m)>\underline{a}(M, \bar{A})$, that is,

$$
\frac{i}{\pi l y}<1+n \frac{M}{M+\sigma m} .
$$

If (107) does not hold, the borrower does not obtain credit if $a \in[\bar{a}(m), \underline{a}(M, \bar{A})]$ and he obtains credit if $a>\underline{a}(M, \bar{A})$.

\subsection{2 $a<\bar{a}(M)$ : collateral poor firms}

If $a<\bar{a}(M)$, the borrower can always choose a repayment scheme that induces the lender to implement the action that allows innovation. This implies that the borrower always chooses relational funding. The borrower chooses a repayment scheme that induces innovation if and only if

$$
(1-\sigma) \pi(1+n) y+\sigma[\pi y+(1-\pi) \sigma M p a]>\pi y+(1-\pi) M p,
$$

that is

$$
a<a^{*} \equiv \frac{\pi n y}{(1+\sigma)(1-\pi) M p} .
$$

There are two cases to consider, $\bar{a}(M) \leq a^{*}$ and $\bar{a}(M)>a^{*}$.

$\bar{a}(M) \leq a^{*}:$ preferred outcome is $(M, A)$ for all $a<\bar{a}(M) \quad$ We can rewrite $\bar{a}(M) \leq a^{*}$ as

$$
\frac{i}{\pi l y} \geq 1+n-\frac{n \sigma}{l(1+\sigma)}
$$

Credit is never available if $\underline{a}(M, A) \geq \bar{a}(M)$, that is,

$$
\frac{i}{\pi l y} \geq 1+\frac{n}{1+\sigma} .
$$

Note that, if (111) holds, the borrower is also not able to obtain credit if the outcome is $(M, \bar{A})$. This is so because (111) implies $\underline{a}(M, \bar{A}) \geq \underline{a}(M, A)$.

Assume now that (111) does not hold. In this case, the borrower does not obtain credit for all $a \leq \underline{a}(M, A)$, but he obtains credit for all $a \in(\underline{a}(M, A), \bar{a}(M))$. As above, if the borrower does not obtain credit when the outcome is $(M, A)$, he does not obtain credit when the outcome is $(M, \bar{A})$. The reason is that, whenever (111) does not hold, we have $\underline{a}(M, \bar{A})>\bar{a}(M)$.

$\bar{a}(M)>a^{*}:$ preferred outcome is $(M, A)$ if $a<a^{*}$, and it is $(M, \bar{A})$ if $a \in\left[a^{*}, \bar{a}(M)\right) \quad$ We can rewrite $\bar{a}(M)>a^{*}$ as

$$
\frac{i}{\pi l y}<1+n-\frac{n \sigma}{l(1+\sigma)}
$$

Note that (112) is non-empty if and only if

$$
l>\frac{1}{1+\sigma}
$$

First, observe that $a^{*}>\underline{a}(M, \bar{A})$ if and only if

$$
\frac{i}{\pi l y}<1+\frac{n}{36^{l(1+\sigma)}} .
$$


This inequality is always satisfied since (112) implies (114). Thus, there is always credit in region (112) if the outcome is $(M, \bar{A})$. Now, observe that $a^{*}>\underline{a}(M, A)$ if and only if

$$
\frac{i}{\pi l y}<1+n-n \sigma+\frac{n \sigma^{2}}{l(1+\sigma)}
$$

which is always true since (112) implies (115). Thus, the outcome is $(M, A)$ if $a \in\left[\underline{a}(M, A), a^{*}\right)$, but there is no credit if $a<\underline{a}(M, A)$. As above, it is straightforward to show that, if $(112)$ holds, then $\underline{a}(M, \bar{A})>\underline{a}(M, A)$. In words, if the borrower does not obtain credit under $(M, A)$, he also does not obtain credit under $(M, \bar{A})$.

\subsection{3 $a \in[\bar{a}(M), \bar{a}(m))$ : firms with intermediate collateral values}

Now, if a borrower wants to have the opportunity to innovate, he must choose transactional funding. This implies that, if there is credit, the equilibrium outcome is either $(m, A)$ or $(M, \bar{A})$. We obtain that $(m, A)$ is preferable to $(M, \bar{A})$ if and only if

$$
(1-\sigma) \pi(1+n) y+\sigma[\pi y+(1-\pi) \sigma m p a]>\pi y+(1-\pi) M p,
$$

that is,

$$
a<\widehat{a} \equiv \frac{(1-\sigma) \pi n y}{(1-\pi) p\left(M-\sigma^{2} m\right)} .
$$

We decompose the case of intermediate collateral values in regions of parameters:

$$
\begin{aligned}
& \mathrm{A} \quad: \quad 1+n-n \sigma<\frac{i}{\pi l y} \leq 1+n-n \sigma \frac{(1-\sigma) M}{l\left(M-\sigma^{2} m\right)} \\
& \mathrm{B} \quad: \quad 1+n-n \sigma \frac{(1-\sigma) M}{l\left(M-\sigma^{2} m\right)}<\frac{i}{\pi l y}<1+n-n \sigma \frac{(1-\sigma) m}{l\left(M-\sigma^{2} m\right)} \\
& \mathrm{C} \quad: \quad 1+n-n \sigma \frac{(1-\sigma) m}{l\left(M-\sigma^{2} m\right)} \leq \frac{i}{\pi l y}<1+n .
\end{aligned}
$$

In region $\mathrm{A}, \widehat{a} \leq \bar{a}(M)$ and, if there is credit, the equilibrium outcome is always $(M, \bar{A})$. In region $\mathrm{C}$, $\widehat{a} \geq \bar{a}(m)$ and, if there is credit, the equilibrium outcome is always $(m, A)$. Finally, in region $\mathrm{B}$, if there is credit, the equilibrium outcome is $(m, A)$ if $a \in[\bar{a}(M), \widehat{a})$, and $(M, \bar{A})$ if $a \in[\widehat{a}, \bar{a}(m))$.

Region A : $\widehat{a} \leq \bar{a}(M)$ First, region A is non-empty if and only if $l>\frac{(1-\sigma) M}{M-\sigma^{2} m}$, which we assume in this subsection. We need to check the conditions under which credit is available.

(i) Credit is always available: This requires $\bar{a}(M)>\underline{a}(M, \bar{A})$, that is,

$$
\frac{i}{\pi l y}<1+\frac{n}{1+\sigma}
$$

If $l \in\left(\frac{(1-\sigma) M}{M-\sigma^{2} m}, \frac{(1-\sigma)(M+\sigma M)}{M-\sigma^{2} m}\right]$, this is always the case in region A. If $l>\frac{(1-\sigma)(M+\sigma M)}{M-\sigma^{2} m}$, this is the case if and only if

$$
\frac{i}{\pi l y} \in\left(1+n-n \sigma, 1+\frac{n}{1+\sigma}\right)
$$

(ii) Credit is never available: This requires $\bar{a}(m) \leq \underline{a}(M, \bar{A})$, that is,

$$
\frac{i}{\pi l y} \geq 1+n \frac{M}{M+\sigma m} \text {. }
$$


If $l>\frac{M}{m} \frac{(1-\sigma)(M+\sigma m)}{M-\sigma^{2} m}$, this is the case if and only if

$$
\frac{i}{\pi l y} \in\left[1+n \frac{M}{M+\sigma m}, 1+n-n \sigma \frac{(1-\sigma) M}{l\left(M-\sigma^{2} m\right)}\right] .
$$

If, instead, $l \in\left(\frac{(1-\sigma) M}{M-\sigma^{2} m}, \frac{M}{m} \frac{(1-\sigma)(M+\sigma m)}{M-\sigma^{2} m}\right]$, then it is never the case.

We need to check whether credit would be available under $(m, A)$ conditional on it never being available under $(M, \bar{A})$. A necessary condition is $\bar{a}(m)>\underline{a}(m, A)$, that is,

$$
\frac{i}{\pi l y}<1+\frac{n}{1+\sigma}
$$

which can never be satisfied if (123) holds.

(iii) Partial credit: If $l>\frac{M}{m} \frac{(1-\sigma)(M+\sigma m)}{M-\sigma^{2} m}$, then in the interval

$$
\frac{i}{\pi l y} \in\left[1+\frac{n}{1+\sigma}, 1+n \frac{M}{M+\sigma m}\right)
$$

there is partial credit, that is, the borrower does not obtain credit if $a \in[\bar{a}(M), \underline{a}(M, \bar{A})]$, but he obtains credit if $a \in(\underline{a}(M, \bar{A}), \bar{a}(m))$. The same is true in the interval

$$
\frac{i}{\pi l y} \in\left[1+\frac{n}{1+\sigma}, 1+n-n \sigma \frac{(1-\sigma) M}{l\left(M-\sigma^{2} m\right)}\right]
$$

as long as $l \in\left(\frac{(1-\sigma)(M+\sigma M)}{M-\sigma^{2} m}, \frac{M}{m} \frac{(1-\sigma)(M+\sigma m)}{M-\sigma^{2} m}\right]$. If $l \in\left(\frac{(1-\sigma) M}{M-\sigma^{2} m}, \frac{(1-\sigma)(M+\sigma M)}{M-\sigma^{2} m}\right]$, the region with partial credit is empty.

As above, we need to check whether credit would be available under $(m, A)$ when it is not available under $(M, \bar{A})$. A necessary condition is $\underline{a}(M, \bar{A})>\underline{a}(m, A)$, that is,

$$
\frac{i}{\pi l y}<1+n \frac{(1-\sigma) M}{M-\sigma^{2} m}
$$

which can never be satisfied under either (126) or (127).

Region B : $\widehat{a} \in\left((\bar{a}(M), \bar{a}(m))\right.$ First, region B is non-empty if and only if $l>\frac{(1-\sigma) m}{M-\sigma^{2} m}$, which we assume in this subsection.

(B.1) $a \in[\widehat{a}, \bar{a}(m)):(M, \bar{A})$ dominates $(m, A)$

(i) Credit is always available: This requires $\underline{a}(M, \bar{A})<\widehat{a}$, that is,

$$
\frac{i}{\pi l y} \leq 1+n \frac{M(1-\sigma)}{l\left(M-\sigma^{2} m\right)} .
$$

If $l \in\left(\frac{(1-\sigma) m}{M-\sigma^{2} m}, \frac{(1-\sigma)(M+\sigma m)}{M-\sigma^{2} m}\right]$, this is always the case. If $l>\frac{(1-\sigma)(M+\sigma M)}{M-\sigma^{2} m}$, this is never the case. In turn, if $l \in\left(\frac{(1-\sigma)(M+\sigma m)}{M-\sigma^{2} m}, \frac{(1-\sigma)(M+\sigma M)}{M-\sigma^{2} m}\right]$, this is the case if and only if

$$
\frac{i}{\pi l y} \in\left(1+n-n \sigma \frac{(1-\sigma) M}{l\left(M-\sigma^{2} m\right)}, 1+n \frac{M(1-\sigma)}{l\left(M-\sigma^{2} m\right)}\right] .
$$

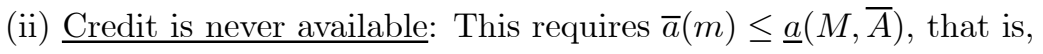

$$
\frac{i}{\pi l y} \geq 1+\underset{38}{M+\sigma m} \text {. }
$$


If $l \geq \frac{M}{m} \frac{(1-\sigma)(M+\sigma m)}{M-\sigma^{2} m}$, this is always the case. If $l \in\left(\frac{(1-\sigma) m}{M-\sigma^{2} m}, \frac{(1-\sigma)(M+\sigma m)}{M-\sigma^{2} m}\right]$, this is never the case. In turn, if $l \in\left(\frac{(1-\sigma)(M+\sigma m)}{M-\sigma^{2} m}, \frac{M}{m} \frac{(1-\sigma)(M+\sigma m)}{M-\sigma^{2} m}\right)$, this is the case if and only if

$$
\frac{i}{\pi l y} \in\left(1+n \frac{M}{M+\sigma m}, 1+n-n \sigma \frac{(1-\sigma) m}{l\left(M-\sigma^{2} m\right)}\right] .
$$

We need to check whether credit would be available under $(m, A)$ conditional on it never being available under $(M, \bar{A})$. A necessary condition is $\bar{a}(m)>\underline{a}(m, A)$, that is,

$$
\frac{i}{\pi l y}<1+\frac{n}{1+\sigma}
$$

which can never be satisfied under (131).

(iii) Partial credit: If $l \in\left(\frac{(1-\sigma)(M+\sigma M)}{M-\sigma^{2} m}, \frac{M}{m} \frac{(1-\sigma)(M+\sigma m)}{M-\sigma^{2} m}\right)$, then in the interval

$$
\frac{i}{\pi l y} \in\left(1+n-n \sigma \frac{(1-\sigma) M}{l\left(M-\sigma^{2} m\right)}, 1+n \frac{M}{M+\sigma m}\right]
$$

the borrower does not obtain credit if $a \in[\widehat{a}, \underline{a}(M, \bar{A})]$, but he obtains credit if $a \in(\underline{a}(M, \bar{A}), \bar{a}(m))$. The same is true in the interval

$$
\frac{i}{\pi l y} \in\left(1+n \frac{M(1-\sigma)}{l\left(M-\sigma^{2} m\right)}, 1+n \frac{M}{M+\sigma m}\right]
$$

as long as $l \in\left(\frac{(1-\sigma)(M+\sigma m)}{M-\sigma^{2} m}, \frac{(1-\sigma)(M+\sigma M)}{M-\sigma^{2} m}\right]$. If either $l \geq \frac{M}{m} \frac{(1-\sigma)(M+\sigma m)}{M-\sigma^{2} m}$ or $l \in\left(\frac{(1-\sigma) m}{M-\sigma^{2} m}, \frac{(1-\sigma)(M+\sigma m)}{M-\sigma^{2} m}\right]$, the region with partial credit is empty.

Again, it remains to check whether credit would be available under $(m, A)$ when it is not available under $(M, \bar{A})$. A necessary condition is $\underline{a}(M, \bar{A})>\underline{a}(m, A)$, that is,

$$
\frac{i}{\pi l y}<1+n \frac{(1-\sigma) M}{M-\sigma^{2} m}
$$

which can never be satisfied under either (134) or (135).

(B.2) $a \in(\bar{a}(M), \widehat{a}):(m, A)$ dominates $(M, \bar{A})$

(i) Credit is always available: This requires $\underline{a}(m, A)<\bar{a}(M)$, that is,

$$
\frac{i}{\pi l y}<1+n-n \sigma \frac{M}{M+\sigma m}
$$

If $l \in\left(\frac{(1-\sigma) m}{M-\sigma^{2} m}, \frac{m}{M} \frac{(1-\sigma)(M+\sigma m)}{M-\sigma^{2} m}\right]$, then this is always the case. If $l \geq \frac{(1-\sigma)(M+\sigma m)}{M-\sigma^{2} m}$, this is never the case. In turn, if $l \in\left(\frac{m}{M} \frac{(1-\sigma)(M+\sigma m)}{M-\sigma^{2} m}, \frac{(1-\sigma)(M+\sigma m)}{M-\sigma^{2} m}\right)$, this is the case if and only if

$$
\frac{i}{\pi l y} \in\left(1+n-n \sigma \frac{(1-\sigma) M}{l\left(M-\sigma^{2} m\right)}, 1+n-n \sigma \frac{M}{M+\sigma m}\right) .
$$

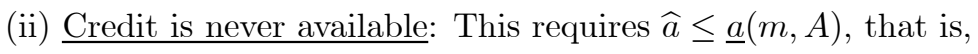

$$
\frac{i}{\pi l y} \geq 1+n-n \sigma+\frac{n \sigma(1-\sigma) \sigma m}{l\left(M-\sigma^{2} m\right)}
$$


If $l \geq \frac{(1-\sigma)(M+\sigma m)}{M-\sigma^{2} m}$, this is always the case. If $l \in\left(\frac{(1-\sigma) m}{M-\sigma^{2} m}, \frac{(1-\sigma)(m+\sigma m)}{M-\sigma^{2} m}\right]$, this is never the case. In turn, if $l \in\left(\frac{(1-\sigma)(m+\sigma m)}{M-\sigma^{2} m}, \frac{(1-\sigma)(M+\sigma m)}{M-\sigma^{2} m}\right)$, this is the case if and only if

$$
\frac{i}{\pi l y} \in\left(1+n-n \sigma+\frac{n \sigma(1-\sigma) \sigma m}{l\left(M-\sigma^{2} m\right)}, 1+n-n \sigma \frac{(1-\sigma) m}{l\left(M-\sigma^{2} m\right)}\right) .
$$

We need to check whether credit is available if the outcome is $(M, \bar{A})$ conditional on it being never available under $(m, A)$. Note that $\underline{a}(M, \bar{A})<\widehat{a}$ implies

$$
\frac{i}{\pi l y} \leq 1+n \frac{M(1-\sigma)}{l\left(M-\sigma^{2} m\right)}
$$

Since

$$
1+n \frac{M(1-\sigma)}{l\left(M-\sigma^{2} m\right)}>1+n-n \sigma+\frac{n \sigma(1-\sigma) \sigma m}{l\left(M-\sigma^{2} m\right)}
$$

we obtain that whenever

$$
\frac{i}{\pi l y} \in\left(1+n-n \sigma+\frac{n \sigma(1-\sigma) \sigma m}{l\left(M-\sigma^{2} m\right)}, 1+n \frac{M(1-\sigma)}{l\left(M-\sigma^{2} m\right)}\right),
$$

credit is not available under $(M, \bar{A})$ for all $a \in(\bar{a}(M), \underline{a}(M, \bar{A})]$ but it is available under $(M, \bar{A})$ for all $a \in(\underline{a}(M, \bar{A}), \widehat{a})$. In the complementary region, i.e., the region given by

$$
\frac{i}{\pi l y} \in\left[1+n \frac{M(1-\sigma)}{l\left(M-\sigma^{2} m\right)}, 1+n-n \sigma \frac{1}{l} \frac{(1-\sigma) m}{M-\sigma^{2} m}\right),
$$

credit is never available under $(M, \bar{A})$.

(iii) Partial credit: If $l \in\left(\frac{m}{M} \frac{(1-\sigma)(M+\sigma m)}{M-\sigma^{2} m}, \frac{(1-\sigma)(m+\sigma m)}{M-\sigma^{2} m}\right)$, then in the interval

$$
\frac{i}{\pi l y} \in\left[1+n-n \sigma \frac{M}{M+\sigma m}, 1+n-n \sigma \frac{1}{l} \frac{(1-\sigma) m}{M-\sigma^{2} m}\right),
$$

the borrower does not obtain credit if $a \in[\bar{a}(M), \underline{a}(m, A)]$, but he obtains credit if $a \in(\underline{a}(m, A), \widehat{a})$. The same is true in the interval

$$
\frac{i}{\pi l y} \in\left[1+n-n \sigma \frac{M}{M+\sigma m}, 1+n-n \sigma+\frac{n \sigma(1-\sigma) \sigma m}{l\left(M-\sigma^{2} m\right)}\right]
$$

as long as $l \in\left(\frac{(1-\sigma)(m+\sigma m)}{M-\sigma^{2} m}, \frac{(1-\sigma)(M+\sigma m)}{M-\sigma^{2} m}\right)$.

Once more, we need to check whether credit is available if the outcome is $(M, \bar{A})$ conditional on it being not available under $(m, A)$. Note that $\bar{a}(M)>\underline{a}(M, \bar{A})$ if and only if

$$
\frac{i}{\pi l y}<1+\frac{n}{1+\sigma} \text {. }
$$

If $l<\frac{(1-\sigma)(m+\sigma m)}{M-\sigma^{2} m}$, this condition is always verified in (145). If $l>\frac{(1-\sigma)(m+\sigma m)}{M-\sigma^{2} m}$, this condition is always verified in (146). Thus, all borrowers obtain credit under $(M, \bar{A})$ in the region with partial credit.

Region $\mathbf{C}: \widehat{a} \geq \bar{a}(m)$ First, note that region $\mathrm{C}$ is never empty.

(i) Credit is always available: This requires $\underline{a}(m, A)<\bar{a}(M)$, that is,

$$
\frac{i}{\pi l y}<1+n-n \sigma \frac{M}{M+\sigma m} .
$$


If $l \geq \frac{m}{M} \frac{(1-\sigma)(M+\sigma m)}{M-\sigma^{2} m}$, then this is never the case. If, instead, $l<\frac{m}{M} \frac{(1-\sigma)(M+\sigma m)}{M-\sigma^{2} m}$, then this is the case if and only if

$$
\frac{i}{\pi l y} \in\left(1+n-n \sigma \frac{1}{l} \frac{(1-\sigma) m}{M-\sigma^{2} m}, 1+n-n \sigma \frac{M}{M+\sigma m}\right) .
$$

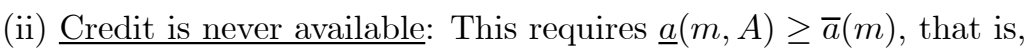

$$
\frac{i}{\pi l y} \geq 1+\frac{n}{1+\sigma} \text {. }
$$

If $l>\frac{(1-\sigma)(m+\sigma m)}{M-\sigma^{2} m}$, this is always the case. If, instead, $l \leq \frac{(1-\sigma)(m+\sigma m)}{M-\sigma^{2} m}$, then this is the case if and only if

$$
\frac{i}{\pi l y} \in\left(1+\frac{n}{1+\sigma}, 1+n\right) \text {. }
$$

We need to check whether the outcome $(M, \bar{A})$ would allow access to some credit. Note that $\underline{a}(M, \bar{A})>\bar{a}(M)$ if and only if

$$
\frac{i}{\pi l y}<1+\frac{n}{1+\sigma},
$$

which is inconsistent with (150). Thus, some borrowers who do not obtain credit under $(m, A)$ will also be denied credit under $(M, \bar{A})$. Now, note that $\underline{a}(M, \bar{A})<\bar{a}(m)$ holds if and only if

$$
\frac{i}{\pi l y}<1+n \frac{M}{M+\sigma m} \text {. }
$$

Thus, if

$$
\frac{i}{\pi l y} \in\left(1+\frac{n}{1+\sigma}, 1+n \frac{M}{M+\sigma m}\right)
$$

and the outcome is $(M, \bar{A})$, the borrower obtains credit if $a \in(\underline{a}(M, \bar{A}), \bar{a}(m))$, but does not obtain credit if $a \in[\bar{a}(M), \underline{a}(M, \bar{A})]$. In turn, if (154) does not hold, i.e., if

$$
\frac{i}{\pi l y}>\left(1+n \frac{M}{M+\sigma m}, 1+n\right)
$$

credit is never available, irrespective of the outcome.

(iii) Partial credit: If $l<\frac{m}{M} \frac{(1-\sigma)(M+\sigma m)}{M-\sigma^{2} m}$, then in the interval

$$
\frac{i}{\pi l y} \in\left[1+n-n \sigma \frac{M}{M+\sigma m}, 1+\frac{n}{1+\sigma}\right]
$$

the borrower does not obtain credit for all $a \in[\bar{a}(M), \underline{a}(M, \bar{A})]$, but he obtains credit for all $a \in(\underline{a}(M, \bar{A}), \bar{a}(m))$. The same is true in the interval

$$
\frac{i}{\pi l y} \in\left(1+n-n \sigma \frac{1}{l} \frac{(1-\sigma) m}{M-\sigma^{2} m}, 1+\frac{n}{1+\sigma}\right],
$$

as long as $l \in\left[\frac{m}{M} \frac{(1-\sigma)(M+\sigma m)}{M-\sigma^{2} m}, \frac{(1-\sigma)(m+\sigma m)}{M-\sigma^{2} m}\right]$. Clearly, if $l>\frac{(1-\sigma)(m+\sigma m)}{M-\sigma^{2} m}$, the region with partial credit is empty.

It remains to check whether the borrower gains access to credit if he chooses $(M, \bar{A})$ when $a \in[\bar{a}(M), \underline{a}(m, A))$. Note that $\underline{a}(M, \bar{A}) \leq \bar{a}(M)$ if and only if

$$
\frac{i}{\pi l y} \leq 1+\frac{n}{1+\sigma}
$$

which is always the case under either (156) or (157). Thus, all borrowers who do not obtain credit under $(m, A)$ do so under $(M, \bar{A})$. This concludes the complete characterization of outcomes in equilibrium. 